\title{
Rock-and-soil avalanches: Theory and simulation
}

\author{
Alfredo Taboada ${ }^{1,2}$ and Nicolas Estrada ${ }^{1,2}$ \\ Received 20 May 2008; revised 11 November 2008; accepted 18 February 2009; published 22 July 2009.
}

[1] We present a 2-D Contact Dynamics discrete element model for simulating initiation and motion of rock avalanches, integrating hillslope geometry, Mohr-Coulomb rock behavior, pore pressure before avalanche triggering, and avalanche trigger. Avalanche motion is modeled as a dense granular flow of dry frictional and cohesive particles. On the basis of granular physics and shear experiments, we review some of the theories for the unexpectedly long runout of rock avalanches. Different causes are evoked, according to the strength (strong or weak) of the slip surface relative to the bulk. "Mechanical fluidization" and "acoustic fluidization" theories state that agitation of rock particles reduces frictional strength, increasing runout. Conversely, granular mechanics suggests that, as "shear-strain" rate increases, granular material becomes more agitated, more dissipative, and more resistant. Another theory states that dynamic fragmentation of clasts creates an isotropic pressure that drives longer runout. In contrast, granular mechanics suggests that fragmentation may induce fluidization and strengthening of the granular material, while particle size reduction (among others) induces weakening of the granular flow and enhances long runout. Runout is also enhanced for column-like rock masses collapsing from steep hillslopes. Long runout may also be linked to thermal weakening mechanisms at the slip surface (e.g., thermal pressurization, and shear melting), which may lower drastically the shear strength. The model is illustrated with a hypothetical example of a rain-triggered avalanche, mobilizing shallowly dipping layers. Several phases are identified, including slope failure, avalanche triggering resulting from slip weakening, and avalanche motion in which rocks are folded and sheared.

Citation: Taboada, A., and N. Estrada (2009), Rock-and-soil avalanches: Theory and simulation, J. Geophys. Res., 114, F03004, doi:10.1029/2008JF001072.

\section{Introduction}

[2] Large bedrock landslides may evolve into avalanches, which are among the largest surface processes observed in hillslope areas [Cruden and Varnes, 1996; Densmore et al., 1997]. Understanding avalanches is a concern among geoscientists and engineers, because of their impact on landscape morphology and geological hazard estimates. Numerical simulations can provide a valuable insight into the physical mechanisms underlying avalanche triggering and motion for these purposes.

[3] To simulate landslides that evolve into avalanches, two central questions must be dealt with: when does the sliding mass become unstable, and how does the unstable mass move to its new equilibrium position. The first question, the stability and deformation of the landslide before failure, is traditionally studied using "limit equilibrium" and "finite element" methods. However, both methods become inappropriate when the landslide becomes unstable. Thus, a different philosophy must be adopted to

\footnotetext{
${ }^{1}$ UMR 5243, Géosciences Montpellier, Université Montpellier II, INSU, CNRS, Montpellier, France.

${ }^{2}$ ADEPT, International Associated Laboratory, NSC, CNRS, Rennes, France.

Copyright 2009 by the American Geophysical Union. 0148-0227/09/2008JF001072
}

study the second question: the motion of the sliding mass. To study the motion, either of two different approaches are usually adopted: the continuum approach, and the discontinuum approach.

[4] The continuum approach, developed using the formalism first introduced by Saint-Venant in 1850, considers the sliding mass as a continuum material, and it is generally based on a depth-integrated version of the Navier-Stokes equation [e.g., Savage and Hutter, 1991; Douady et al., 2002]. This approach treats the avalanche as a single-phase flow, and, in addition to the depth-integrated conservation equations, a depth-averaged rheology must be assigned to the sliding mass (e.g., Bingham fluid rheology). However, for most geomaterials, the rheology under dynamic conditions is not known a priori, nor can it be measured experimentally, and therefore, the rheological choice must be conjectural.

[5] The discontinuum approach considers the sliding mass as a collection of distinct elements that interact through contact forces, and is based in the time integration of the equations of motion of individual particles. In the discontinuum approach, the sliding mass can be heterogeneous, since different "species" of particles can be used in the same model. Here, the user does not choose the rheology of the bulk, but, rather, the behavior of the contacts between particles; the rheology of the bulk results 
naturally from "contact-scale" interactions. However, the rheology of the bulk might not be representative of the real material, in particular when the real material is not sufficiently "granular." Two well known methods developed with this philosophy are "Molecular Dynamics" [Cundall and Stack, 1979] and "Contact Dynamics".

[6] In spite of the diversity of methods to simulate landslides and avalanches, and of the differences between them, several fundamental questions remain open; for instance, how does a landslide evolve into an avalanche, and what are the mechanisms underlying the unexpectedly long-runout distance of avalanches? The goals of this paper are as follows: (1) to revisit, in the light of results from granular dynamics and high-velocity shear experiments, some of the possible causes for the long runout of avalanches and (2) to present a numerical model for the simulation of granular avalanches that integrates the initiation and motion phases.

[7] In our model, the sliding mass is approximated by a collection of particles simulated by means of Contact Dynamics. We adopt the two following simplifying assumptions: (1) Prior to avalanche initiation (i.e., in quasistatic conditions), rocks and soils may be modeled as cohesive granular material subjected to gravity and pore pressure forces. (2) During the avalanche motion (i.e., in dynamic conditions), the material may be modeled as a dense flow of dry frictional and cohesive particles.

[8] Using granular physics, we briefly review and discuss some of the theories that try to explain the long runout of rock avalanches in terms of the dynamic behavior of the bulk. Additionally, we show that thermal weakening postulated for earthquake faults may be potentially active along the slip surface of rock avalanches. Thus, we suggest that long runout may result from several causes, which are linked either to granular processes in the bulk or to dynamic weakening mechanisms at the slip surface.

[9] Our conceptual model is illustrated by simulating a hypothetical example of a rain-triggered avalanche, which mobilizes gently dipping strata. The numerical experiment raises the water table until the tilted layers become unstable. This permits analysis of the whole avalanche sequence from initiation to deposition in terms of deformation mechanisms in the granular assembly and forces applied at the boundaries. The model also simulates the macroscopic structure of the deposit.

[10] The outline of the paper is as follows: the "discrete element" approach is presented in section 2; the conceptual model for simulating rock avalanches is presented in section 3; section 4 analyses weakening mechanisms operating from initiation of motion onward; the hypothetical example of an avalanche triggered by rain is presented in section 5; the analysis of the simulation is presented in section 6; and last, the main findings are summarized and discussed in section 7 .

\section{Discrete Element Methods}

\section{1. "Soft Particle" Versus "Hard Particle" Models}

[11] Discrete element methods (this is, methods that use the discontinuum approach) can be classified in two main groups: those that use "soft particles" and those that use "hard particles." Soft particle methods (e.g., Molecular Dynamics [Cundall and Stack, 1979]) consider the particles as being deformable at the contacts. To simulate contact deformation, particles are allowed to overlap one another, and contact forces are calculated as a function of the small overlap distances. In order to follow the evolution of contact deformations, and to ensure numerical stability, these methods require very small time steps. Such high resolution cannot be avoided when the elastic deformation of the particles is of primary importance (e.g., when studying transmission of sound waves through a granular material); nevertheless, for simulating processes involving very large plastic strains, which is generally the case with surface processes, these methods are time consuming.

[12] Hard particle methods (e.g., Contact Dynamics) consider particles as being perfectly rigid. Here, contact forces are calculated in order to verify certain kinematical constraints, such as the noninterpenetrability of particles and Coulomb friction. Because of the different formulation of the equations of motion and contact laws, these methods do not require following the evolution of contact deformations in order to maintain numerical stability, do not introduce elastic repulsion potentials, nor damping coefficients, and do not need to smooth (i.e., regularize) the contact laws (e.g., the Coulomb friction law). Thus, these methods are faster than the soft particle methods and are well adapted to study granular materials at large plastic strains (e.g., beyond the scale of elastic deformations).

\subsection{Contact Dynamics Method}

[13] The simulations presented in this work are based on the Contact Dynamics (CD) method, which is suitable for simulating the mechanical behavior of large assemblies of rigid particles. The CD method was initiated by Moreau [1988, 1994, 1999] and Jean [1995, 1999] using concepts from nonsmooth (i.e., nonregular) dynamics and convex analysis. Since then, it has been used to study the behavior of granular materials [Moreau, 1993; Radjaï et al., 1996; Daudon et al., 1997; Chevoir et al., 2001; Azéma et al., 2007; Staron and Hinch, 2007]. For cohesionless particles, the CD method is based on the implicit time integration of the equations of motion, the nonsmooth formulation of impenetrability and Coulomb friction, and the dissipation of energy by means of a restitution coefficient. An introduction to the CD method is given by Taboada et al. [2005b] and Pöschel and Schwager [2005].

[14] In order to simulate surface processes, we introduce cohesion in the framework of the CD method; the idea is to mimic cohesion by cementation, as observed in soils and rocks. Our model incorporates the following two features: (1) The strength of a cohesive contact depends on three contact laws defining local thresholds for applied forces (traction and shear) and torque. (2) The kinetics of contact gain and loss are governed by a creation distance $\Delta$ and an elliptic zone of weak extension to represent the cementing material. In all the simulations presented in this paper the restitution coefficient was set to zero, as is the case for most geomaterials.

\subsubsection{Strength of a Cohesive Bond}

[15] The strength of a cohesive bond depends on three contact laws defining the threshold forces and torques that it can resist. The three contact laws represent the three relative degrees of freedom between particles: separation, which implies relative velocity in the direction normal to the 

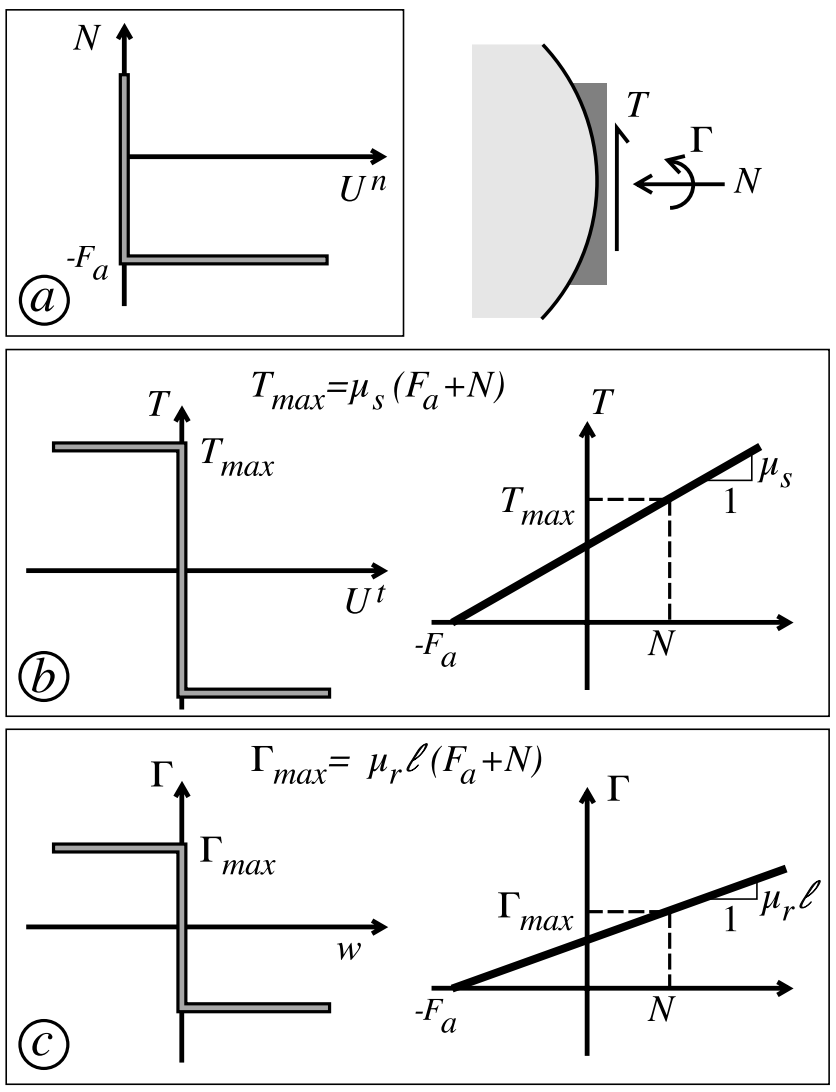

Figure 1. Graphs defining the contact laws of a cohesive bond. (a) Signorini graph illustrating the relation between the relative normal velocity and the normal contact force. (b) Mohr-Coulomb friction law relating the relative tangential velocity and the shear force; the shear rupture envelope is shown on the right. (c) Relation between the relative angular velocity and the torque at a cohesive contact; the torque failure envelope is shown on the right.

contact; sliding, which implies relative velocity in the direction tangential to the contact; and rolling, which implies relative angular velocity between particles.

[16] The normal interaction is defined by the so-called Signorini graph, relating the relative normal velocity $U^{n}$ and the normal force $N$ (Figure 1a). The Signorini graph expresses the impenetrability of particles through the two following mutually exclusive conditions:

$$
\begin{array}{ccc}
U^{n}=0 & \text { and } & N \geq-F_{a} \\
U^{n}>0 & \text { and } & N=-F_{a},
\end{array}
$$

where we attribute positive values to compressive forces, $U^{n}$ is considered positive when particles move away from each other, and $-F_{a}$ is the largest tensile force that can be supported by a cohesive bond. The tensile threshold $-F_{a}$ is given by

$$
F_{a}=\sigma_{\text {ext }} \ell,
$$

where $\sigma_{\text {ext }}$ is the tensile strength and $\ell$ is the average diameter of the particles in contact.
[17] The tangential interaction is represented by the Coulomb friction law, relating the relative tangential velocity $U^{t}$ and the tangential force $T$ (Figure 1b). The Coulomb friction law implies the three following excluding conditions:

$$
\begin{array}{ll}
U^{t}>0 & \text { and } \quad T=-T_{\max } \\
U^{t}=0 & \text { and } \quad-T_{\max } \leq T \leq T_{\max } \\
U^{t}<0 & \text { and } \quad T=T_{\max }
\end{array}
$$

where $T_{\max }$ is the largest shear force that can be supported by a cohesive bond. The shear threshold $T_{\max }$ is proportional to the normal force $N$ and is given by

$$
T_{\max }=\mu_{s}\left(F_{a}+N\right),
$$

where $\mu_{s}$ is the coefficient of sliding friction.

[18] Full cementation requires also torque transmission at the contacts. By analogy with the Coulomb friction law, the rolling friction law relates the relative angular velocity $w$ and the contact torque $\Gamma$ (Figure 1c). The rolling friction law implies the three following mutually exclusive conditions:

$$
\begin{aligned}
& w>0 \quad \text { and } \quad \Gamma=-\Gamma_{\max } \\
& w=0 \quad \text { and } \quad-\Gamma_{\max } \leq \Gamma \leq \Gamma_{\max } \\
& w<0 \quad \text { and } \quad \Gamma=\Gamma_{\max },
\end{aligned}
$$

where $\Gamma_{\max }$ is the largest torque that can be supported by a cohesive bond. The torque threshold $\Gamma_{\max }$ is proportional to the normal force $N$ and is given by

$$
\Gamma_{\max }=\mu_{r} \ell\left(F_{a}+N\right),
$$

where $\mu_{r}$ is the coefficient of rolling friction. Rolling friction is a consequence of flexure at the scale of the cohesive bond; the parameter $\mu_{r}$ can be interpreted as the arm of the torque that opposes flexure, normalized by the mean diameter of the particles in contact $\ell$. Thus, in our model, the strength of a cohesive bond depends on three parameters: $\sigma_{\text {ext }}, \mu_{s}$, and $\mu_{r}$.

\subsubsection{Kinetics of Contact Loss and Gain}

[19] Cohesive bonds are modeled initially between all grains such that the gap $\delta$ (i.e., the distance between their two closest material points $P_{i}$ and $P_{j}$ ) is below $\Delta$ (Figure 2a). A cohesive bond persists as long as the point $P_{j}$ is located inside a small elliptical zone fixed to disk $i$ (Figure 2b). Contact rupture leads to an irreversible loss of tensile strength (debonding) and the contact turns to purely frictional behavior.

[20] The creation distance $\Delta$ and the size of the elliptic zone (i.e., the size of its axes $\epsilon_{n}$ and $\epsilon_{t}$ ) are small compared to the size of the particles. These three lengths control the scale of strain softening and the energy dissipated during the rupture of a cohesive bond.

\section{Simulation of Rock-and-Soil Avalanches Using Granular Materials}

[21] The term "rock-and-soil avalanche" encompasses a large variety of surface processes that share the following common features [Heim, 1932; Legros, 2002; Strom, 2006]: (1) high velocity (between 5 and $300 \mathrm{~m} / \mathrm{s}$ ), (2) large volumes (typically $>10^{5} \mathrm{~m}^{3}$ ), (3) long runout (generally 


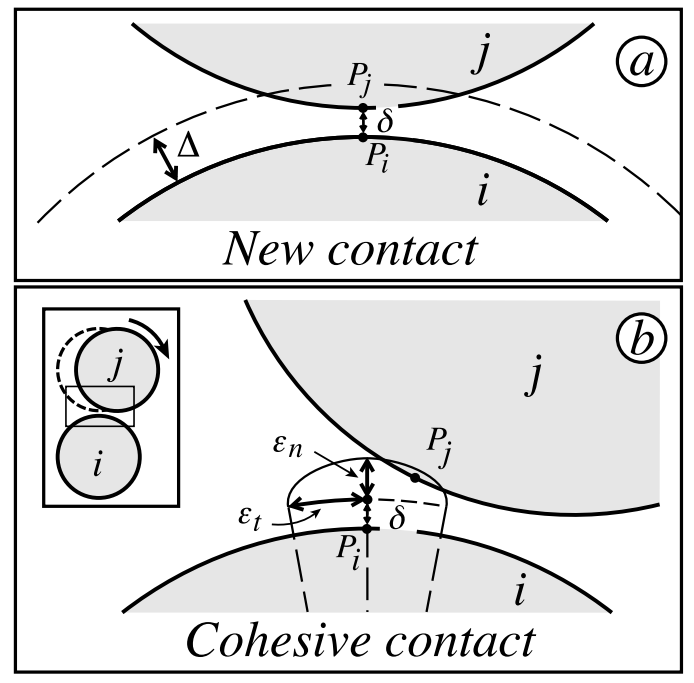

Figure 2. (a) Criterion for the creation of a new contact between two grains ( $i$ and $j$ ) defined according to a threshold distance $\Delta$. (b) Small elliptical zone in which cohesive bonding persists.

defined in terms of the travel angle or fahrböschung), (4) comminution of the rock-and-soil mass (although this condition is not always fulfilled), and (5) lack of mixing of different lithologies from the slope failure to the resultant debris deposit (although this condition is not always fulfilled).

[22] Various scale laws have been proposed relating some of these features. For example, it is universally found that the travel angle decreases as avalanche volume increases. This correlation suggests that there is some mechanism that reduces friction as the size of the avalanche increases. Given the similarities between rock-and-soil avalanches and "rock avalanches," both terms will be used synonymously.

[23] Rock avalanches may originate, among others, from large rockslides or cliff falls, or from the collapse of steep hillslopes [e.g., Cruden and Varnes, 1996; McSaveney and Davies, 2006]. Regardless of the geological setting, the initial bedrock failure occurs along a surface of rupture, which is generally located along a preexisting weak zone such as a bedding plane, a weak layer, or a master joint. During initiation, a surface of rupture may be subjected to shearing or tensile stresses, depending on the initial displacement field of the unstable mass.

[24] Schematically, all these processes involve mobilization of rock and soil materials (i.e., the bulk) over an interface termed the slip surface. The slip surface is composed of the surface of rupture and an adjoining surface located downslope, termed the surface of separation, which corresponds to the part of the original ground surface overlain by the avalanche deposit.

[25] The dynamics and deformation of a rock avalanche are partly controlled by the relative strengths of the slip surface and the bulk. If the slip surface is much stronger than the bulk, then the shear strain will be mostly distributed within the bulk; conversely, if the slip surface is much weaker than the bulk, then the shear displacement will mostly concentrate along the slip surface. If the substrate is weaker than the mobilized materials then the slip surface may shift from the original ground surface to another interface located beneath.
[26] The following sections describe our conceptual model for the simulation of rock-and-soil avalanches by means of a discrete element approach. The procedure for the construction of a granular hillslope model is presented, as well as the hypotheses concerning the behavior of both the bulk and the boundaries. The role of pore water pressure in the bulk, prior to and during the avalanche, is also considered.

\subsection{Hillslope Granular Model}

[27] We assume that the initial state of a hillslope can be simulated as a 2-D granular model constructed from a geological cross section (Figure 3 ). The orientation of the section parallels the direction of mobilization of the poten-

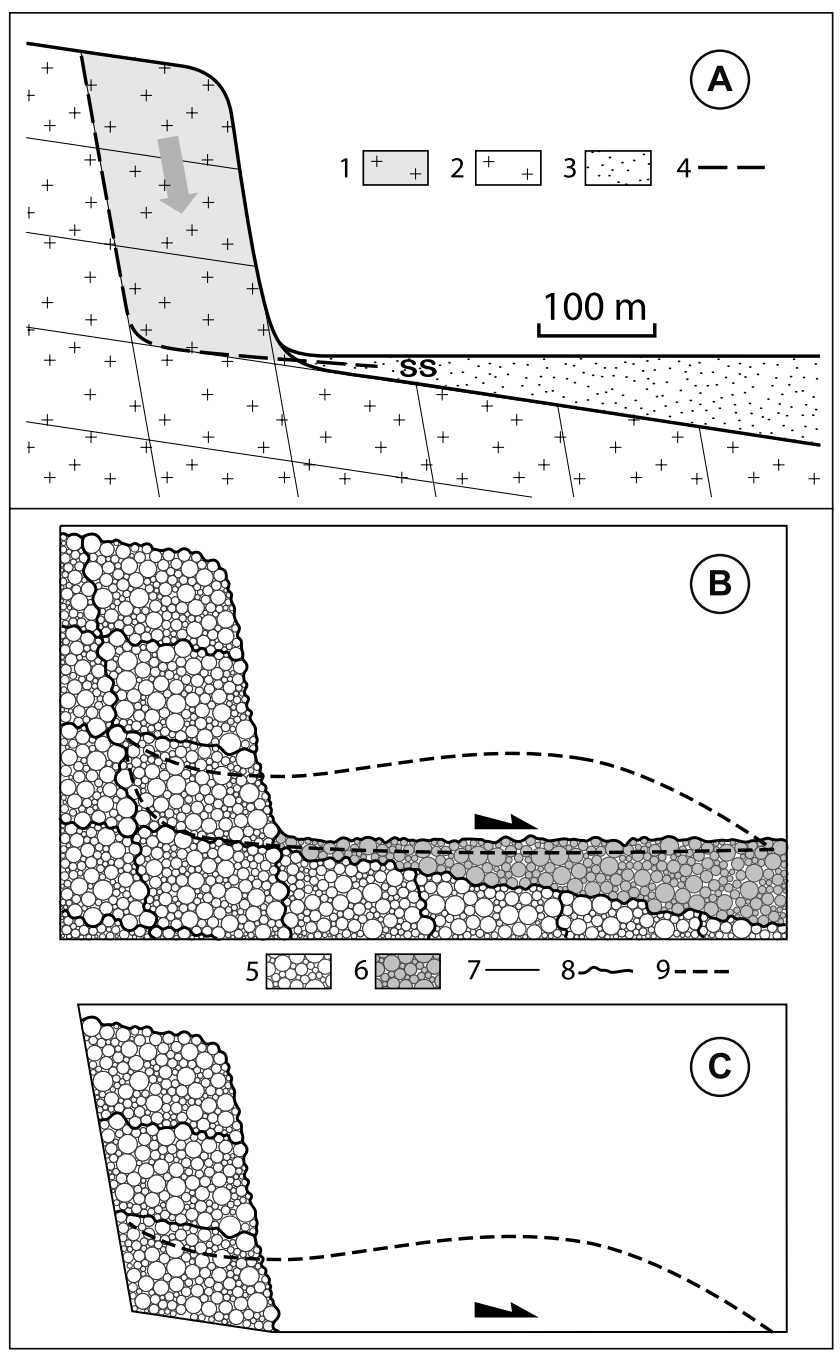

Figure 3. Granular models constructed from a geological cross section across a potentially unstable hillslope. (a) Cross section; (b) granular model in which the slip surface is located along granular interfaces; (c) granular model in which boundaries are defined along the presumed slip surface. Here 1, potentially unstable rocks; 2, stable bedrock; 3, weak sediments; 4, potential slip surface; 5, granular material modeling rock; 6, granular material modeling sediments; 7 , boundaries of the granular model; 8 , boundaries of the granular domains; 9 , avalanche debris. 
tial landslide. The geological structure is constructed by dividing the hillslope area into granular domains (e.g., layers) with varying properties.

[28] The granular model is bounded by a set of lines representing the physical boundaries of the system. In principle, the boundaries may be located in the rock volume underlying the slip surface, in which case the slip surface is located along a granular interface (Figure 3b); this choice allows for erosion and entrainment of granular material located beneath the initial slip surface. However, boundaries can also be defined along the presumed slip surface of the landslide (Figure 3c); this choice reduces the number of particles in the system, and facilitates the introduction of specific shear stress laws along the slip surface during sliding motion.

[29] The modeling of the slip surface by a set of line segments is consistent with field observations indicating that this interface is very narrow compared with the average avalanche thickness. For example, in the Vaiont rockslide in Italy and the Jiufengershan rock-and-soil avalanche in Taiwan the thickness of the slip surface was submillimetric to centimetric [e.g., Chang et al., 2005a; Veveakis et al., 2007]. These are similar to estimated thicknesses of shear bands in rocks and soils, which roughly involve between 10 and 200 average size particles [Vardoulakis, 2002a; Rempel and Rice, 2006].

[30] Inception of motion is controlled by the slip surface geometry, the slip surface strength, and a triggering mechanism. Some of the most common natural landslide triggers are intense rainfall, rapid snowmelt, water level change, earthquake shaking, and foothill erosion. These triggers can be simulated in a granular model by considering simplifying hypotheses. For example: the first three triggers involve pore water pressure and groundwater flow, which can be simulated by applying buoyancy and seepage forces on particles (see section 3.4); earthquake shaking may be introduced by imposing a velocity field along the bounding lines; and foothill erosion may be introduced by eliminating particles according to given criteria (e.g., particles located within a mobile window that simulates erosion).

\subsection{Bulk}

\subsubsection{Strength}

[31] Quasistatic strengths of rocks and soils may be characterized by the Mohr-Coulomb behavior law [Goodman, 1989]. Mohr-Coulomb behavior is defined by an angle of internal friction and cohesion, which are specified for both maximum and residual strengths. These parameters control the shear strengths of geomaterials, at initial failure and for large strains, respectively. Similarly, granular materials deformed in quasistatic conditions also show Mohr-Coulomb behavior at macroscopic scale [e.g., Taboada et al., 2006]. However, the macroscopic strength parameters (i.e., those of the particle set), are essentially different from the microscopic strength parameters (i.e., those of the contacts). Thus, the strength parameters at the contact-scale $\mu_{s}, \mu_{r}$, and $\sigma_{\text {ext }}$, must be carefully selected in order to match the strength properties of rock materials in the hillslope. This calibration process is a common exercise when dealing with granular materials, and it consists of performing numerical shear tests on a granular sample. This parametric analysis allows investigation of the nontrivial relationship between micro- scopic and macroscopic strength properties [Estrada et al., 2008].

[32] Sliding along discontinuities that cut across the hillslope granular model (e.g., interlayer sliding) is controlled by the shear strength along interfaces between adjacent granular domains (e.g., the bedding planes). Strong interfaces are simulated by defining high frictional and cohesive contacts, whereas weak interfaces are simulated by defining low-friction and cohesionless contacts. Note that the macroscopic friction along a granular interface cannot be set to zero, even if the contacts between particles are frictionless. For disordered granular packings, the lowest macroscopic friction angle lies between $4^{\circ}$ and $7^{\circ}$ [Taboada et al., 2006]. This minimum friction angle results from the inherent roughness of granular shear zones, which tends to resist sliding even for frictionless particles. The dynamic behavior issues of rocks and soils involved in an avalanche are discussed in section 4.

\subsubsection{Scaling Principles for Granular Materials}

[33] Simulation of rock avalanches as granular material raises the following questions concerning the scaling of the model: (1) What kind of particle assembly should be selected to simulate geomaterials? (2) What is the physical meaning of the particles in the model, in particular, during motion? (3) What is the effect of changing particle sizes?

[34] As regards the first question, two simple rules may be proposed for generating granular assemblies that are adequate for simulating geologic materials. Firstly, the distribution of particle sizes should be wide and even (i.e., well graded), in order to introduce disorder and avoid "crystallization" of the particle set and to account for geometrical similarity with particle-size distributions in a rock avalanche. Disordered granular assemblies have a relatively isotropic behavior, as observed, to a certain extent, in many rocks and soils. Secondly, the pore volume should be low. Many geomaterials are dense granular assemblies and exhibit brittle behavior, tending to localize deformation along dilatant shear bands.

[35] As regards the second question, particles in a granular model do not represent true particles in a rock avalanche, which are generally much smaller. The scale of particles in the model is selected according to the scale of structures and processes to be simulated; these determine the macroscopic resolution scale. In granular physics, the smallest particle set with homogeneous mechanical properties is named the "representative elementary volume" (REV). For an accurate simulation, the REV should be smaller than the macroscopic resolution scale. This criterion imposes a maximum size to the particles in the model. In practice, the scale of particles should be at least 1-2 orders of magnitude smaller than the macroscopic resolution scale.

[36] As regards the third question, we may reasonably expect that, for homothetic particle sets that share common strength properties, the simulation results at the macroscopic resolution scale are similar. This scaling attribute is linked with the definition of the contact strength thresholds, which involves a length scale given by the average diameter of the particles in contact (see section 2.2).

\subsection{Boundaries}

[37] The strength parameters at line-disk contacts must be calibrated in order to match the macroscopic strength 


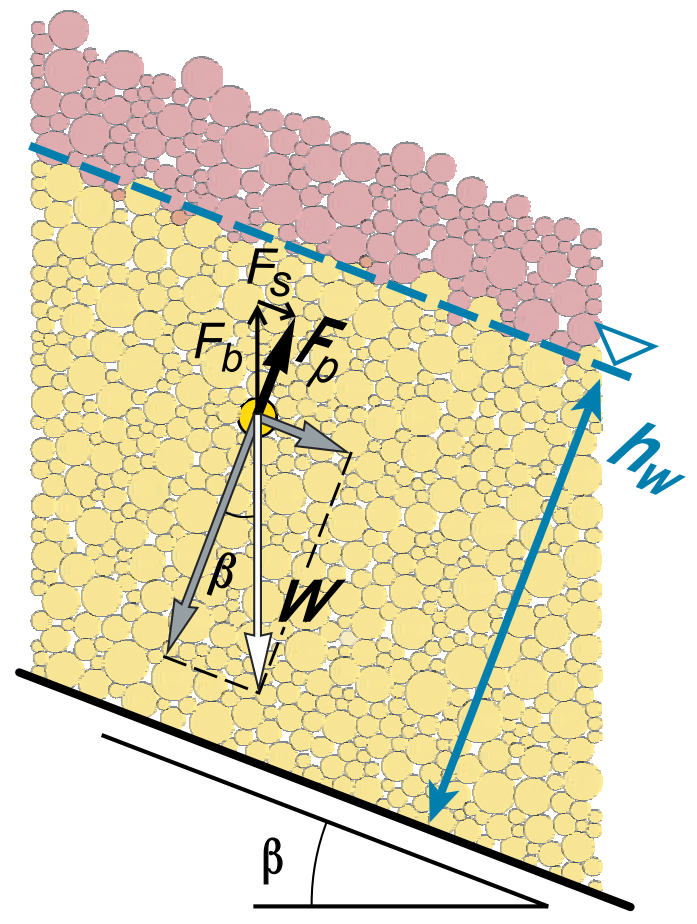

Figure 4. Volume forces applied at the center of mass of a particle located below water table. $W$ is the particle weight, $F_{p}$ is the net force exerted by the pore fluid on the particle, $F_{b}$ and $F_{s}$ are the buoyancy and seepage forces exerted by the pore fluid on the particle, $\beta$ is the slope of the granular layers, and $h_{w}$ is the water table height above sliding plane.

defined for each line segment. The line segments that bound granular materials are intrinsically smooth (i.e., there are no asperities as in granular shear bands that cut across the bulk). Thus, the relations that link contact-scale and macroscopic friction and adhesion along a line segment are straightforward. On the one hand, because of the absence of asperities, the macroscopic friction coefficient is the same as the sliding friction coefficient at line-particle contacts. On the other hand, the contact and macroscopic adhesions are roughly proportional to each other, since contact adhesion is proportional to particle diameter. For dense granular materials, both adhesion parameters are approximately equal.

[38] In dynamic conditions, the shear strength along an interface may lower during the sliding process, according to specific weakening mechanisms. Weakening behavior can be incorporated in the model, because the shear strength along a smooth interface can be set to any value including zero, in contrast to the shear strength of the bulk. These issues are discussed in detail in section 4.3.

\subsection{Pore Water Pressure Prior to Avalanche Triggering}

[39] Fluids in permeable hillslope rocks have a major influence on slope stability, since they modify the internal stresses within the hillslope. It is generally assumed that the pore water pressure carries part of the normal stress, and consequently the effective normal stress and the shear strength decrease as the groundwater table rises. These stress variations can occur while the hillslope is in quasistatic conditions, and they may induce landsliding.
[40] The pore water pressure distribution in a hillslope can be determined using numerical or analytical groundwater flow solutions [e.g., Bear, 1988]. The effect of pore water pressure can be introduced in the granular model by considering the combined effect of buoyancy and seepage forces applied on the 2-D disks [e.g., Mourgues and Cobbold, 2003]. Buoyancy forces result from submersion of a particle under fluid, while seepage forces are due to frictional drag exerted by the pore fluid moving through the interconnected pore network [Mitchell and Soga, 2005]. In this approach, each disk is subjected to an additional volume force $F_{p}$ that is proportional to both the pore water pressure gradient $\nabla p$ and the volume of the particle $V$ (Figure 4)

$$
F_{p}=\nabla p V
$$

This force results from integrating the pore water pressure over the particle surface, and it is applied at the center of mass of the particle. Thus, it should be interpreted as a contact force proportional to the volume of the particle. The buoyancy force is represented by an updipping vector, while the seepage force is oriented in the average flow direction (e.g., downslope). Thus, the seepage force may contribute to the destabilization of the hillslope.

\subsection{Pore Water During Avalanche Motion}

[41] Dynamic shearing and fragmentation of materials during a rock-and-soil avalanche induces dilatancy [e.g., Pollet and Schneider, 2004]. For instance, an average expansion value of $\sim 20 \%$ was calculated for the Jiufengershan rock-and-soil avalanche, whose debris deposit is up to $100 \mathrm{~m}$ thick [Chang et al., 2005a].

[42] As a general rule, dilatancy reduces pore water pressure in the bulk. Dilatancy increases during avalanche motion as rock material is sheared and fragmented. The newly created voids in between rock fragments are not completely filled with groundwater and the material may be considered as unsaturated. In our conceptual model, we assume that the effect of pore water pressure in the bulk is negligible during rapid avalanche motion, and accordingly, the avalanche may be modeled as a dense granular flow of dry frictional and cohesive particles.

\section{Long Runout of Rock Avalanches}

[43] While a large number of theories have been advanced to explain the long runout of large rock avalanches, the subject is still a matter of debate [Hungr, 2006]. These theories may be classified into two groups, depending on the physical mechanism that is put forward. In the first group, the long runout is explained in terms of physical processes occurring within granular rock material in the bulk, such as mechanical fluidization, acoustic fluidization, or dynamic fragmentation [e.g., Campbell, 1989; Collins and Melosh, 2003; McSaveney and Davies, 2006]. In this case, the avalanche may propagate over a rough surface, without a need to invoke lowered basal friction. Conversely, in the second group, the long runout is inferred to be the consequence of mobilization over a slip surface with very low shear strength. Several theories have been proposed to explain the causes of the lowering of the shear strength, 


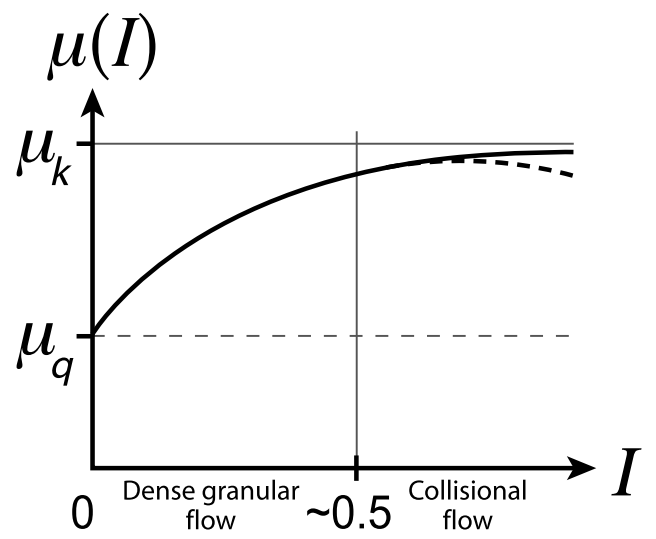

Figure 5. Sketch illustrating friction coefficient $\mu$ as a function of the inertial parameter $I$.

most of which invoke specific mechanisms of dynamic weakening (i.e., the shear strength decreases as the displaced mass accelerates downslope).

[44] Detailed analysis underlying the unexpectedly long runout of rock avalanches is outside the scope of this paper. Nevertheless, in this section we discuss the reliability of some of the main theories that try to explain long runout, in the light of granular mechanics, thermomechanical models, and high-velocity friction experiments [e.g., Di Toro et al., 2004; Pouliquen et al., 2005; Rice, 2006]. Firstly, we describe the rheology of dense granular flows in terms of an empirical friction law based on experimental and numerical simulations. Next, we analyze the hypotheses underlying the mechanical fluidization, the acoustic fluidization, and the dynamic fragmentation theories, among others. Last, we review mechanisms that may be at the origin of dynamic slip surface weakening, and, in particular, those involving frictional or viscous heating.

\subsection{Rheology of Dense Granular Flows}

[45] The shear strength of dense granular flows has been a matter of study for many years, using a great variety of devices and loading conditions [Groupement de Recherche Milieux Divises, 2004]. Experimental and theoretical results show that the coefficient of friction of a sheared granular material varies with the mean "agitation" of its particles. The coefficient of friction $\mu$ can be expressed as a function of a dimensionless inertial parameter $I$. Thus,

$$
\mu(I)=\tau / P
$$

where $\tau$ is the shear stress and $P$ is the confining pressure. The inertial parameter $I$ is defined as

$$
I=\dot{\gamma} d \sqrt{\rho / P}
$$

where $\dot{\gamma}$ is the shear strain rate, $d$ is the average particle diameter, and $\rho$ is the particle density [Da Cruz et al., 2004; Iordanoff and Khonsari, 2004; Rognon et al., 2007]. The inertial parameter is interpreted as the ratio between a microscopic timescale $(d \sqrt{\rho / P})$ and a macroscopic timescale related to the strain rate $(1 / \dot{\gamma})$. The microscopic timescale represents the average time for microscopic rearrangements of unstable particles that pass over the crest of other particles and fall into the next hollow space, as a result of average contact forces (i.e., confining pressure). The macroscopic timescale represents the average time for shearing the granular material a unit value.

[46] The following three granular flow regimes may be distinguished according to the value of the inertial parameter $I$ : (1) Low values of $I\left(\lesssim 10^{-3}\right)$ correspond to quasistatic flows since macroscopic shear is slow compared to microscopic rearrangements. (2) Intermediate values of $I\left(10^{-3} \lesssim\right.$ $I \lesssim 0.5)$ correspond to dense granular flows in which the granular material flows like a liquid, showing large force networks and collective motions. (3) Higher values of $I$ $(\gtrsim 0.5)$ correspond to collisional granular flows in which the medium behaves as a dissipative gas, where particle interactions are dominated by binary collisions.

[47] Numerical and experimental results suggest that natural granular flows including rock-and-soil avalanches occur in the dense regime (e.g., with solid concentrations well above $50 \%$ by volume), and not in the collisional regime [Campbell, 2006]. Natural granular flows apparently cannot generate large enough shear rates and granular temperature to support the material at small concentrations.

[48] The coefficient of friction $\mu$ increases asymptotically as a function of the inertial parameter $I$, from a quasistatic value $\mu_{q}$ to a maximum value $\mu_{k}$ for the collisional flow regime (i.e., when the granular material is strongly agitated) (Figure 5) [Forterre and Pouliquen, 2008]. Actually, the friction coefficient $\mu$ measures the ability of a granular material to dissipate mechanical energy by means of inelastic collisions and contact friction. Nevertheless, according to numerical simulations, the friction coefficient may eventually decrease when reaching the collisional flow regime.

[49] In practice, the friction coefficient $\mu(I)$ of rock material during an avalanche cannot be measured. Additionally, the inertial parameter $I$ presumably varies in space and time as a consequence of comminution, heterogeneous shearing, and stress variations. Thus, the function $\mu(I)$ for a granular model cannot be calibrated with respect to field data, yet it is inherent to sheared granular materials (i.e., the friction coefficient $\mu$ in the dynamic regime is always empirical).

\subsection{Theories on the Dynamic Behavior of the Bulk}

\subsubsection{Mechanical Fluidization}

[50] Mechanical fluidization is one of the early theories that were proposed to explain the long runout of rock avalanches in terms of bulk granular behavior [e.g., Davies, 1982; Campbell, 1989]. It is based on two hypotheses concerning the kinematics and the dynamics of granular flows. The hypothesis on kinematics assumes that the bulk of material rides on a thin layer of highly agitated particles of low concentration. The hypothesis on dynamics assumes that the friction angle of a rapidly shearing dry granular material will decrease as a function of increasing velocity (i.e., agitation generates dynamic interactions and fluidization of the granular material, forcing particles away from each other and reducing frictional stress).

[51] The hypothesis on kinematics is partly consistent with some numerical and experimental results on granular flows (see section 4.2.4); a transient basal layer of agitated particles subjected to intense shear may be present near the 
front of granular flows, although the layer flow regime is apparently dense and not collisional. Conversely, the hypothesis on dynamics is not consistent with the empirical law presented in the previous section, which suggests that the friction coefficient in a granular flow increases as a function of the shear strain rate $\dot{\gamma}$. The key point is that fluidization or agitation resulting from rapid shearing may induce strengthening of granular materials, and not weakening as required by this theory. Mechanical fluidization was challenged by early experimental and numerical results on granular flows: flume experiments from Hungr and Morgenstern [1984] suggested that the dynamic friction angle increases with speed, while numerical simulations from [Campbell et al., 1995] suggested that the granular mass is completely shearing and that the apparent friction coefficient is an increasing function of shear rate.

\subsubsection{Acoustic Fluidization}

[52] The acoustic fluidization theory for long runout of rock avalanches is also based on two hypotheses concerning the kinematics and the dynamics of granular flows [Collins and Melosh, 2003]. The hypothesis on kinematics assumes that acoustic waves with wavelengths comparable to avalanche size may propagate through the granular material, generating random vibrations of groups of particles organized into waves; acoustic waves may result from large, high-frequency pressure fluctuations generated during the initial collapse and subsequent flow of a mass of rock debris. The hypothesis on dynamics assumes that pressure fluctuations linked to acoustic waves may locally relieve overburden stresses in a flowing rock mass, reducing the frictional resistance to slip between fragments and facilitating rapid fluid-like flow.

[53] The hypothesis on kinematics is partly consistent with experimental results from vertically vibrated granular systems of macroscopic identical spheres, which show fluidized and crystallized particle layers organized into waves [e.g., Perez et al., 2008]. However, the extremely widely graded particle size distribution in rock avalanches probably generates acoustic waves with varying amplitudes and wavelengths (i.e., white noise), as suggested by sound and seismic frequencies emitted from rock avalanches. Thus, particle motion is probably random, and not well organized into waves of a given wavelength as assumed in this theory. The hypothesis on dynamics is not consistent with the frictional behavior of granular materials since random vibrations are a form of agitation that induces strengthening of the granular material, and not weakening as required by this theory. Moreover, transient pressure fluctuations are dampened rapidly as a result of friction and inelastic collisions. Note that the friction coefficient characterizing shear strength is defined over a macroscopic time interval, which is much greater than the period of a vibrational cycle.

\subsubsection{Dynamic Fragmentation}

[54] The dynamic fragmentation theory proposes a different explanation for the unexpectedly long-runout distance of large rock avalanches [McSaveney and Davies, 2006]. This theory is based on two hypotheses concerning the dynamics of avalanche motion. The first hypothesis assumes that some kinetic energy is "consumed" in clast elastic strain to be released very rapidly upon clast fragmentation, creating an isotropic clast pressure within the avalanche interior, as breaking clasts interact with surrounding clasts. Fragmenting clasts may be considered as a highly agitated granular phase (e.g., "bubbles of heavy vapor") flowing across a moderately persistent network of particles characterized by enduring contacts and a force network (e.g., a "granular liquid"). In other words, a rock avalanche may be imaged as a biphased granular material consisting of a dense granular flow and an interstitial granular gas. The second hypothesis assumes that the pressure from fragmentation resists part of the confining pressure, reducing normal contact forces in the force network, and driving longer runout without a need to invoke a lowered macroscopic friction coefficient.

[55] These hypotheses may be examined in the light of results from granular physics. For this, we recall some general features concerning the distribution of contact forces within shearing granular samples in quasistatic and dense flow regimes. For instance, the larger contact forces in the force network are mainly supported by chain-like groups of particles that are roughly oriented parallel to the major principal stress $\sigma_{1}$, and constitute what is known as the strong network. Conversely, the weak network carries small forces and it is roughly oriented parallel to the minor principal stress $\sigma_{3}$. The principal stress axes are oriented obliquely in relation with the shear direction (e.g., in simple shear they are oriented at $45^{\circ}$ from the shear axis).

[56] To our knowledge dynamic fragmentation of clasts during a rock avalanche has not yet been modeled. However, simulations of shear tests on granular samples performed at low strain rates may give some insights on failure and fragmentation mechanisms in granular flows [e.g., Taboada et al., 2005b]. During shearing, the principal stresses show fluctuations and instabilities that result from reorganizations in the contact force network. The principal stress $\sigma_{1}$ presents cyclic variations, each cycle showing the following three successive phases (Figure 6): a steady stress increment, a short stress drop, and a transient unstable phase characterized by high-frequency fluctuations and high compressional peaks. As explained forward, the granular flow regimes during the three phases may be considered as dense, transitional, and collisional, respectively. The first phase is associated with dilation in the granular mass resulting from rigid body rotations of the strong force chains (i.e., interval $\left[t_{1}, t_{2}\right]$, Figure 6). Conversely, the two subsequent phases are associated with a sharp contraction of the granular material; the highest compressional peaks are produced when contraction reaches a maximum value. The drop in $\sigma_{1}$ results from buckling of the strong force chains and shear failure at the more highly stressed contacts (i.e., time $t_{2}$ ), and the consecutive collapse of nearby particles in the force network. The transient unstable phase results from agitation and multiple collisions between particles in the collapsing zone, suggesting that the granular material is locally fluidized (i.e., time $t_{3}$ ). The failure mechanism of a strong force chain involves the lateral ejection of unstable particles in the chain; the average direction of ejection is perpendicular to the force chains (i.e., parallel to $\sigma_{3}$ ). Expelled particles generate additional agitation in the surrounding area, therefore increasing "granular temperature." The kinetic energy of both collapsing and expelled particles is partly transferred to nearby grains and progressively dissipated by collisions, depending 


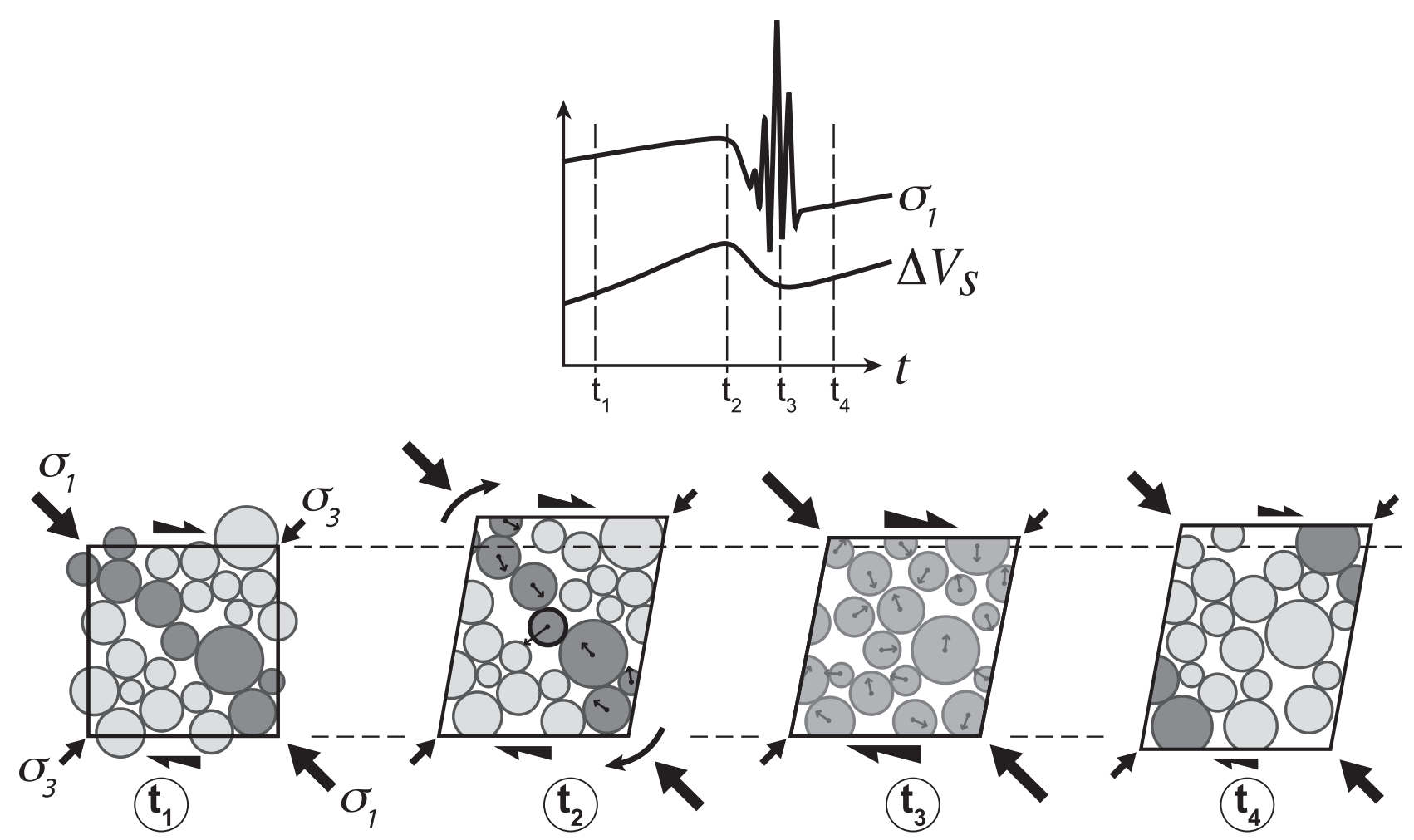

Figure 6. Sketch illustrating cyclic variations in stress $\sigma_{1}$ and volumetric strain $\Delta V_{s}$ in sheared granular material and deformation of a small volume of particles at four successive times (see text for explanation). Here $\left[t_{1}, t_{2}\right]$ is the steady stress increment and dilation of granular material; $t_{2}$ is the failure of strong force chains resulting from lateral ejection of particles and collapse of nearby particles in the force network, entraining stress drop and contraction of granular material; $t_{3}$ is the transient unstable phase resulting from agitation in the collapsing zone, suggesting that granular material is locally fluidized; and $t_{4}$ is the reorganization of contact forces into a new network and beginning of a new stress cycle. Dark and light gray particles belong, respectively, to the strong and weak networks, whereas fluidized particles are indicated by a middle gray shading. Particle velocities during the failure and unstable phases are indicated by arrows, and average stresses are indicated by thick arrows.

on restitution coefficients. After the unstable phase, contact forces reorganize into a new network and a new stress cycle begins (i.e., time $t_{4}$ ). A similar process has been observed in numerical simulations of compaction bands, in which crushing events are well correlated with stress drops and high-frequency stress fluctuations [Marketos and Bolton, 2005].

[57] The following two fundamental questions concerning dynamic fragmentation within dense granular flows are discussed: (1) Where and how does fragmentation occurs? (2) What is the effect of fragmentation on contact forces and shear stress?

[58] As regards the first question, fragmentation of clasts during an avalanche may involve fractures and cracks of varying sizes and orientations, which break up the rock into fragments with a very large size distribution [e.g., McSaveney and Davies, 2006; Carmona et al., 2008]. Tensile splitting fractures may be quite abundant because of the low extensional strength of rock materials; these fractures may be disposed radially around the force chain axis [Taboada et $a l ., 2005 \mathrm{a}]$, and as in Brazilian tests, they may initiate in the clast cores and propagate toward the contact points between clasts. The dynamic or high-energy breakage of clasts pre- sumably induces the collapse of nearby particles in the force network and the ejection of rock fragments in the $\sigma_{3}$ direction, as occurs in shear tests described previously. The threshold for dynamic fragmentation is a function of the mechanical strength of rocks and it imposes an additional constraint on the stability of strong force chains.

[59] As regards the second question, two scenarios may be proposed:

[60] 1. A first scenario consists in supposing that failure of strong chains during shearing is likely to generate similar effects on a granular material regardless of the specific failure mechanism (ejection, fragmentation, or crushing of particles). Under this supposition, it may be inferred that dynamic fragmentation induces cyclic stress and volumetric strain variations as illustrated in Figure 6. In particular, dynamic collisions of collapsing particles and expelled rock fragments may generate a transient unstable phase in the nearby granular material, enhancing agitation and fluidization (i.e., a collisional flow regime). The increase in granular temperature may induce, in turn, an increase in granular pressure and friction coefficient $\mu$, the latter possibly approaching a saturation value $\mu_{k}$ (see Figure 5). According to equation (8), shear stress $\tau$ in a sheared 

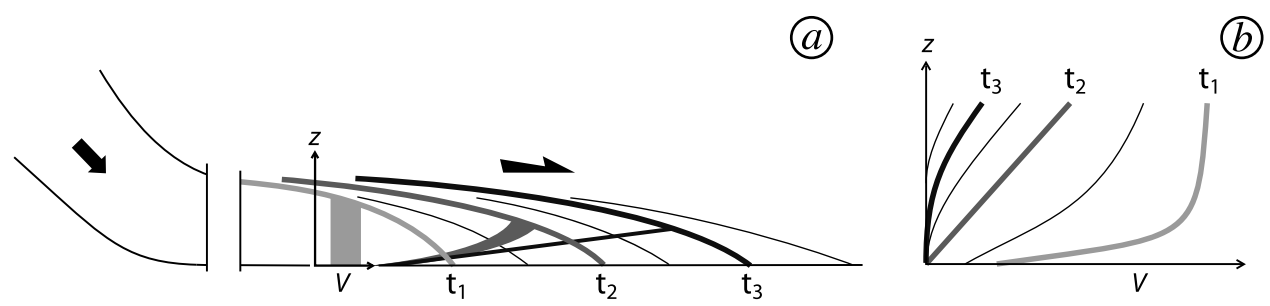

Figure 7. Sketch illustrating the kinematics of a granular avalanche that propagates over a rough horizontal surface. (a) Avalanche surface profiles for three successive times $t_{1}, t_{2}$, and $t_{3}$; (b) velocity profiles for times $t_{1}, t_{2}$, and $t_{3}$, along a vertical axis $z$ fixed to the basal surface. Each velocity profile is characteristic of a flow regime located at a given distance relative to the avalanche tip. Flow regimes may be approximated by a plug flow $\left(t_{1}\right)$, a homogeneous simple shear flow $\left(t_{2}\right)$, and a a two-layered flow $\left(t_{3}\right)$. Cumulative shear strain is illustrated by a vertical column, which is sheared from $t_{1}$ to $t_{3}$.

granular material increases during the transient unstable phase, since $\tau$ is proportional to both pressure and friction. Thus, the unstable phase of dynamic fragmentation is likely to strengthen a sheared granular material and slow down granular flow.

[61] 2. An alternative scenario based on the hypotheses of the dynamic fragmentation theory consists in supposing that fragmentation produces an interstitial granular gas at a given pressure, which flows across a dense granular flow. The coexistence of two granular phases requires that enduring contacts between particles in the force network are strong enough to prevent fluidization of the entire granular material. Note that particle agitation diffuses from the "hightemperature" granular gas toward the "cooler" particles in the force network. The coexistence of a granular liquid and a granular gas is a subject of debate and specific numerical simulations are required to analyze this flow configuration. The stress tensor in a biphased granular material (BGM) may be obtained by adding the stress tensors of each individual phase, provided that each phase involves exclusive particle and contact sets. Thus, the shear stress and confining pressure in a BGM should be somewhere in between the corresponding values for a dense flow and a totally fluidized medium. Accordingly, an interstitial granular gas is likely to strengthen and slow down a dense granular flow, in contradiction with the second hypothesis of the dynamic fragmentation theory.

[62] Contrastingly, there are two conditions linked to dynamic fragmentation that may weaken a granular material in comparison to an equivalent material composed of particles that do not fragment, enhancing long runout; they are as follows: (1) The reduction of particle size due to fragmentation may reduce the coefficient of friction, since the microscopic timescale for particle rearrangements and the inertial parameter are both proportional to average particle size (see section 4.1). (2) The axial stress thresholds for dynamic fragmentation or crushing of particles located in strong force chains may be lower than the thresholds for "buckling" of strong force chains in an equivalent nonfragmenting material.

[63] The second condition is presumably satisfied for a certain proportion of force chains (i.e., those showing particle fragmentation), which depends on confining pressure, geometry of particle chains, and particle shapes and strengths; thus, average deviatoric stresses in a fragmenting granular material (which depend on average forces in the strong force network), ought to be lower than in an equivalent nonfragmenting material, reducing shear stress. Nevertheless, these inferences are not conclusive, since they are based on partial knowledge of mechanics of dynamic fragmentation during a rock avalanche; detailed simulations of rock fragmentation under avalanche shear conditions are required to draw definite conclusions on this theory.

\subsubsection{Insights From Numerical and Experimental} Models

[64] Several features and mechanisms that are at the origin of the long runout of rock avalanches may be put forward, in the light of numerical and experimental models [e.g., Lajeunesse et al., 2005; Staron and Hinch, 2007]. These models consider, in particular, dry granular flows of cohesionless frictional materials that spread over a frictional (rough) horizontal plane. The mechanisms that are identified point out that frictional dissipation is not in contradiction with high mobility and long runout of granular flows.

[65] Long runout is enhanced for column-like rock masses that collapse from steep hillslopes and that show a large aspect ratio at the initial state (i.e., the ratio between height and width). In these circumstances, the spreading dynamics is controlled by the initial geometry of the rock mass rather than by the details of the interactions between rock fragments. For vertical cliffs, the sideways spreading of the grains is initiated by the free-fall dynamics of the rock mass. The stage of vertical acceleration is followed by a "conventional" horizontal granular flow characterized by the propagation of a horizontal mass wave. This mass wave transmits kinetic energy from the rear of the flow where vertical motion prevails, toward the front of the flow where horizontal motion prevails.

[66] Particle motion in a rock-and-soil avalanche varies over both time and space at a variety of scales. Numerical and experimental models of granular avalanches have been used to analyze granular motion in simplified configurations [e.g., Lajeunesse et al., 2005; Friedmann et al., 2006; Staron and Hinch, 2007; Taberlet et al., 2007]. Results from these models suggest that granular motion over a rough horizontal plane may show three flow regimes with differing velocity profiles as a function of depth. The location of the three flow regimes relative to the tip of the granular flow as well as the corresponding velocity profiles are illustrated in Figure 7 . The velocity profiles are defined for successive times, at a vertical axis $z$ fixed to the basal surface. 
[67] The first flow regime may be approximated by a plug flow in which shear strain is concentrated near the bottom of the granular mass (time $t_{1}$, Figure 7). This flow regime is generally observed near the front of the granular flow, mobilizing the whole pile thickness over a thin layer of particles. It results from high-speed advancing grains that overlay the surface of separation.

[68] The second flow regime may be approximated by a homogeneous simple shear flow (i.e., the velocity profile is roughly linear), and it is generally observed upstream of the plug flow at a varying distance of the flow tip (time $t_{2}$, Figure 7). As the granular flow slows down, the location of this flow regime shifts progressively toward the flow tip while the shear rate decreases.

[69] The third flow regime may be approximated by a two-layered flow observed at the rear of the granular flow, characterized by a flowing layer overlying a static layer. The velocity profile is linear in the flowing layer and it shows a lower exponential tail in the static layer (time $t_{3}$, Figure 7). According to the velocity profiles, the granular materials near the base of the flow progressively accrete in a static layer, while the upper grains continue to flow all through the spreading process; as the granular flow slows down, the thickness of the flowing layer decreases.

[70] The shear strain resulting from the three flow regimes is illustrated by a vertical column, which is sheared from $t_{1}$ to $t_{3}$ (Figure 7a). Shear strain migrates progressively from the lowermost part of the granular flow toward the top. Note that the total shear strain is homogeneously distributed within the entire granular mass. Thus, the cumulative horizontal displacement within the granular deposit increases upward, favoring long runout.

[71] The succession between the three flow regimes is closely related to the dissipation rate of kinetic energy in the granular flow. Recall that kinetic energy dissipation rate in the bulk may be approximated by the product of shear stress times shear strain rate, while shear stress is defined as the product of the friction coefficient $\mu(I)$ times the confining pressure $P$. Thus, energy dissipation rate is maximum in the lowermost part of the plug flow since both shear strain rate and shear stress reach peak values. As the lowermost grains slow down, both shearing and energy dissipation migrate upward, giving rise to a roughly homogeneous simple shear flow. Note that the rate of energy dissipation for a linear velocity profile (i.e., constant shear strain rate) increases proportionally with depth (i.e., confining pressure). Thus, particles located in the lower part of the granular flow are slowed down into a static layer while those located in the upper part continue to flow, giving rise to a two-layered flow. This implies that the upper grains preserve kinetic energy until the final stage of granular flow, moving large distances along low-angle trajectories.

\subsection{Theories for Dynamic Weakening of the Slip Surface}

[72] Under certain conditions, mass movements involving sliding along a slip surface (e.g., rockslides), may transform into avalanches [e.g., Chang et al., 2005a]. In this geologic setting, the mechanical behavior and the geometry of the slip surface may determine whether an avalanche triggers or not. We advance the hypothesis that, with few rare exceptions, a large rockslide may transform into an avalanche provided that the slip surface has the following two distinctive features: (1) The slip surface, or at least the surface of rupture, should exhibit dynamic weakening during triggering and motion of the landslide. (2) The geometry of the slip surface should be curvilinear, or alternatively, the surface of separation should be rough. Dynamic weakening of the slip surface may induce mobilization of the bulk at high velocity (e.g., section 6); sliding along a curvilinear slip surface may induce deformation and comminution of the bulk, even if sliding friction is very low (e.g., section 6); and sliding along a rough surface of separation may induce heterogeneous shear flow and comminution of the bulk (e.g., section 4.2.4).

[73] The mechanisms responsible for dynamic weakening of the slip surface may be a function of cumulative slip, sliding velocity and/or temperature. These three distinct mechanical behaviors are known, respectively, as slip, velocity, and thermal weakening.

[74] Shear forces applied at the slip surface, whether frictional or viscous, are dissipative. Dissipative forces transform mechanical energy into heat, plastic deformation, and wave motion. The rate of energy dissipated by frictional or viscous forces is defined, respectively, by the product of slip velocity or shear strain rate times the shear stress. Dynamic weakening implies that the rate of energy dissipation decreases as shear forces become weaker.

[75] Several physical processes have been postulated as potential sources of dynamic weakening along the slip surfaces of landslides and earthquake faults. These mechanisms are thermal pressurization of pore fluid (thermal expansion), degradation of microcontacts by flash heating, thermal decomposition of rock minerals, lubrication by silica gel, and shear melting [e.g., Lin et al., 2001; Di Toro et al., 2004; Rice, 2006; Han et al., 2007]. These all involve heating of the shear zone and hence are plausible only under dynamic conditions (i.e., high slip velocity). Other specific weakening mechanisms such as the lubrication of the slip surface by liquefied saturated soils, may also operate, in particular, along the surface of separation of rock avalanches [e.g., Buss and Heim, 1881; Hungr, 2006]. Soil liquefaction is probably linked to other "state variables" related to vibrations and waves that propagate in the slip surface area.

[76] Dynamic weakening mechanisms involving frictional and viscous heating have mostly been described in the context of seismogenic faults. Thus, in the following sections we review the main assumptions and equations underlying these mechanisms, and we discuss their relevance to the dynamics of rock-and-soil avalanches.

\subsubsection{Thermal Pressurization of Pore Fluid}

[77] This physical process has been proposed as a possible weakening mechanism operating at the slip surface of rapid landslides and earthquake faults [e.g., Sibson, 1973; Lachenbruch, 1980; Rice, 1999; Vardoulakis, 2002b; Chang et al., 2005b; Bizzarri and Cocco, 2006]. It can be observed experimentally in results from frictional heating of saturated materials subjected to shearing at high rates $(1 \mathrm{~m} / \mathrm{s})$ [Wibberley and Shimamoto, 2005]. The origin of pressurization is linked with the difference in thermal behavior between the liquid and solid phases of granular materials present in the shear zone (i.e., pore fluid has a greater thermal expansion coefficient than fault gouge). Thus, pore 
fluid within a narrow shear zone tends to expand at a higher rate than the solid skeleton; if the permeability of the sheared materials is low enough $\left(\sim 10^{-19} \mathrm{~m}^{2}\right)$, then the pore pressure may increase considerably within the shear zone.

[78] The origin of this mechanism can be easily explained by supposing that the shear strength $\tau_{\max }$ of frictional granular materials within the slip surface is defined in terms of the Terzhagi's effective stress theory (i.e., $\tau_{\max }=\mu_{s}\left(\sigma_{n}-\right.$ $p)=\mu_{s} \sigma_{n}^{\prime}$, where $p$ is the pore pressure, $\sigma_{n}$ is the normal stress, and $\sigma_{n}^{\prime}$ is the effective normal stress). Thus, an increase in the pore pressure induces a decrease in the shear strength along the shear zone by reducing the effective normal stress. In theory, this mechanism would reduce the shear strength to a negligible value if the pore pressure approached the applied normal stress.

[79] Simple numerical models have been proposed to quantify the effects of thermal pressurization on the temperature and the shear strength within a shear zone [e.g., Rice, 2006]. These models are based on two simplified conservation equations coupled to thermal and fluid transport equations for the shear zone and adjacent rocks. The first conservation equation is an energy balance as specified by the first law of thermodynamics

$$
-\partial q_{h} / \partial y+\tau \dot{\gamma}=\rho c \partial T / \partial t
$$

where $q_{h}$ is the heat flux in the $y$ direction (perpendicular to the shear zone), $\rho$ is the density, $c$ is the heat capacity per unit mass, $T$ is the temperature, and $t$ is the time. This balance supposes that heat production may be approximated by work done by the shear stress per unit time $(\tau \dot{\gamma})$. Equation (10) states that the change in temperature is proportional to the difference between heat production and heat flux per unit volume. The second conservation equation specifies the conservation of fluid mass within the shear zone

$$
\partial m / \partial t=-\partial q_{f} / \partial y
$$

where $m$ is the mass of fluid per unit volume of bulk material and $q_{f}$ is the fluid mass flux in the $y$ direction. This equation states that, in a small control volume, the fluid mass flux is compensated by the local change in the mass of fluid.

[80] The thermal and mass transport equations are specified by Fourier's and Darcy's laws, respectively

$$
q_{h}=-K(\partial T / \partial y) \quad q_{f}=\left(-\rho_{f} k / \eta\right)(\partial p / \partial y)
$$

where $K$ is the thermal conductivity, $k$ is the permeability, $\rho_{f}$ is the fluid density, and $\eta$ is the dynamic viscosity of the fluid. The evolution of the temperature and the pore pressure in the shear zone is governed by the porothermoplastic properties of both the solid and the fluid phases. Both temperature and pressure in the shear zone evolve according to well known diffusion laws. The spatiotemporal evolutions of the diffusion processes are controlled, respectively, by the thermal and the hydraulic diffusivity [Rempel and Rice, 2006]

$$
\alpha_{t h}=K / \rho c \quad \alpha_{h y}=k / \eta \beta
$$

where $\beta$ is the storage capacity of the solid skeleton.
[81] The solution of these equations is sensitive to parameters such as the permeability and the thickness of the shear zone. In particular, dynamic weakening is enhanced for narrow shear zones with very low permeability. In the case of earthquake faults, the thickness of the shear zone is assumed to be millimetric to submillimetric and the permeability of fault gouge is supposed to be very low $\left(k \sim 10^{-20} \mathrm{~m}^{2}\right)$. The critical slip distance for thermal pressurization in earthquake fault gouges typically ranges between a few millimeters and $5 \mathrm{~cm}$. Analytical solutions for extreme conditions (e.g., adiabatic, undrained deformation or slip on a zero thickness plane) indicate that the shear strength decreases exponentially to zero as a function of slip. This set of equations is valid as long as the temperature of the fluid remains within the liquid stability range, which increases with pressure. The relation between pressure and temperature at the evaporation threshold for water is given approximately by the following equation [Vardoulakis, 2002a]

$$
p_{e v} \approx 62 T_{K} e^{-4650 / T_{K}}
$$

where $T_{K}$ is the absolute temperature $\left({ }^{\circ} \mathrm{K}\right)$ and $p_{e v}$ is the vapor pressure in $\mathrm{MPa}$. For example, at a temperature of $220^{\circ} \mathrm{C}$, the vapor pressure roughly corresponds to the total pressure exerted by a $100 \mathrm{~m}$ column of rocks. Note that this temperature is well below the melting onset for crustal rocks.

[82] An example of a landslide that may have involved thermal pressurization is the disastrous Vaiont rockslide (northern Italy, 1963), which mobilized $\sim 0.27 \mathrm{~km}^{3}$ of rocks along a clay-rich water-saturated slip surface [Veveakis et al., 2007, and references therein]. According to model results, a complete loss of shear strength along the slip surface could be achieved in $2-3 \mathrm{~s}$ and $\sim 5 \mathrm{~m}$ slip, with the temperature during thermal pressurization rising from $\sim 36^{\circ} \mathrm{C}$ to $\sim 70^{\circ} \mathrm{C}$ (well below the evaporation threshold).

[83] Thermal pressurization is possibly most significant during avalanche initiation, and it may decrease as pressurized liquids or gasses escape from the slip surface into nearby fractures and cracks. At this point, the friction coefficient along the slip surface may increase giving rise to additional heat production that, in turn, may trigger other thermal weakening mechanisms.

\subsubsection{Degradation of Microcontacts by Flash Heating}

[84] This mechanism was put forward as a relevant weakening process in crustal earthquake faults [Rice, 1999], and we suggest that it may also operate at the slip surface of thick rock-and-soil avalanches. Flash heating induces weakening at highly stressed frictional microcontacts during rapid slip, supposedly reducing the friction coefficient [Rice, 2006]. The main assumption is that the local shear strength $\tau_{c}$ at asperity contacts degrades with increasing temperature. The effect of flash heating on friction apparently is more intense at submelting temperatures that are close to the melting temperature of rock minerals (e.g., $900^{\circ} \mathrm{C}$ ). These temperatures may be reached in the vicinity of microcontacts, even though the average temperature in the shear zone is well below the onset of melting. 
[85] Theoretical models suggest that severe thermal weakening occurs if the temperature of an asperity contact reaches a critical weakening temperature $T_{w}$ during its brief lifetime [Rice, 1999]. Asperity contacts persist for a micrometric slip distance $D_{a}$ before they are sheared away [Marone, 1998]. The rise in temperature at asperity contacts is enhanced by increasing the sliding velocity: higher sliding velocities increase the available frictional heat by reducing the brief lifetime of asperities and hence the conductive heat loss (see equation (10)). The weakening temperature $T_{w}$ is attained when the sliding velocity reaches a critical velocity $V_{w}$ given by [Rice, 2006]

$$
V_{w}=\left(\pi \alpha_{t h} / D_{a}\right)\left[\rho c\left(T_{w}-T\right) / \tau_{c}\right]^{2},
$$

where $T$ is the initial temperature at the contact.

[86] Note that the critical velocity $V_{w}$ is independent of the confining pressure and depends exclusively on material properties and temperature rise (i.e., in brittle rock materials $\tau_{c} \approx 0.1 G$, where $G$ is the modulus of elasticity in shear). Considering characteristic parameters for fault gouge $\left(\alpha_{t h}=\right.$ $0.5 \mathrm{~mm}^{2} / \mathrm{s}, D_{a}=5 \mu \mathrm{m}, \rho c=2.7 \mathrm{MPa} /{ }^{\circ} \mathrm{C}, \tau_{c}=3 \mathrm{GPa}$ ), and a temperature rise of $\sim 900^{\circ} \mathrm{C}$, then the critical velocity is $V_{w}=0.2 \mathrm{~m} / \mathrm{s}$. This critical velocity suggests that the degradation of microcontacts by flash heating may operate along the slip surface of a rock avalanche from avalanche triggering onward.

\subsubsection{Thermal Decomposition of Rock Minerals}

[87] Thermal decomposition results from frictional heating of rock minerals within narrow fault zones that are sheared at high velocities (i.e., $1 \mathrm{~m} / \mathrm{s}$ [Han et al., 2007]). Very low friction appears to be associated with flash heating of an ultrafine decomposition product composed of nanometric particles, which exhibit degraded microcontacts. According to experiments on simulated faults, thermal decomposition is concentrated on both sides of the slip surface. The thermal decomposition zone is a few millimeters thick, while the slip surface containing the ultrafine particles is 2 orders of magnitude narrower.

[88] This mechanism has been described in rock avalanches mobilizing carbonate rocks (e.g., calcination of marble to create quicklime [Hewitt, 2009]), yet it can also operate in gouges containing sheet silicate minerals containing hydroxyl groups, because their decomposition temperatures are lower than that for calcite decomposition. For carbonate rocks such as marble, thermal decomposition occurs at an average temperature higher than $550^{\circ} \mathrm{C}$ (peak temperature $950^{\circ} \mathrm{C}$ ), inducing a decrease in the coefficient of friction from 0.6 down to 0.06. Carbonate thermal decomposition occurs at submelting temperatures, as suggested by the absence of glass and amorphous materials in the fault zone. Calcium and Magnesium carbonates do not melt because they thermally decompose below their melting points. Thermal decomposition can be a useful paleothermometer in fault studies, and probably also in landslides.

[89] Thermal decomposition may occur along the slip surface of a rock avalanche from avalanche triggering onward. This mechanism has been observed in shear experiments for a large range of confining pressures (1.1-13.4 MPa), and it induces severe weakening for sliding distances ranging from a few to several meters.

\subsubsection{Lubrication by Silica Gel}

[90] Rapid slip experiments performed on quartz-rich rock samples have been interpreted to suggest that friction may decrease drastically because of the formation of a thin layer of silica gel [Di Toro et al., 2004]. These laboratory experiments were conducted at ambient temperature and humidity, at constant slip velocities, and at a confining pressure $\sigma_{n}=5 \mathrm{MPa}$ (i.e., overburden $\sim 200 \mathrm{~m}$ ). The frictional strength in these experiments is characterized by a transient stage for slip displacements less than $\sim 1 \mathrm{~m}$, followed by steady state behavior. During the transient stage, the frictional strength is slip weakening (i.e., the friction coefficient decreases significantly over $\sim 0.5-1 \mathrm{~m}$ of slip). In contrast, the steady state frictional strength is velocity weakening for slip velocities between 0.03 and $0.1 \mathrm{~m} / \mathrm{s}$ (i.e., the friction coefficient drops from $\sim 0.6$ to 0.2 ). Extrapolation of these results suggested that friction falls toward zero as slip velocity approaches $1 \mathrm{~m} / \mathrm{s}$.

[91] A silica-gel-like material was identified in a layer of ultracomminuted gouge, the production of which appears to require water from the humid atmosphere and probably the presence of amorphous silica. The mechanical behavior of this material may be thixotropic, which means that dynamic weakening is promoted by cumulative slip and sliding velocity. Thixotropic materials are considered as pseudoplastic fluids whose apparent viscosity decreases as a function of shear rate. However, the precise nature of this material is conjectural: the material might also correspond to nanoparticle-sized pieces of silica, which in the presence of humidity have the silica crystal structure terminated by covalent bonding to hydroxyl groups, and then covered by a monomolecular layer of high-density ice (as is the case for all natural quartz). The weakening mechanism for this last material would be uncertain since the frictional strength of gouges consisting of quartz fragments and amorphous silica appears to be higher than measured values.

[92] These experimental results are inferred to suggest that silica gel formation may contribute to the lubrication of the slip surface of quartz-rich rock avalanches during triggering and motion. The experimental protocol shows similarities and differences with respect to typical kinematic and overburden pressure conditions expected at the slip surface. On the one hand, the critical slip distance and velocity for dynamic weakening are both similar to those expected during the early stages of very rapid landslides. On the other hand, the confining pressure, and therefore the rate of frictional heat production, are both greater than those expected for most landslides. More laboratory experiments performed at lower confining pressures, higher velocities, and longer-slip distances, may give new insights into the frictional properties of silica gel in the context of rock avalanches.

\subsubsection{Shear Melting}

[93] Frictional and viscous heating may melt comminuted rock along the slip surface of large landslides [e.g., Erismann, 1979; Masch et al., 1985]. For example, fused materials (pseudotachylytes) have been observed on the slip surface of the Jiufengershan and the Tsao-Ling rock-and-soil avalanches, which are the two largest landslides triggered by the 1999 Chi-Chi Taiwan earthquake [Lin et al., 2001; Chang et al., 2005a, K. J. Chang, personal communication, 2003]. The pseudotachylytes in the Jiufengershan avalanche 
occur as a thin layer on the slip surface and as veins injected into oblique cracks. The thickness of the fused layer ranges from 1 to $10 \mathrm{~mm}$. These pseudotachylytes contain typical textures of fused material such as vesicles, glassy matrices and flow structures. The glass content determined by powder X-ray diffraction analysis is as much as $50 \mathrm{wt} \%$. Estimated temperatures inferred for the formation of the pseudotachylytes range between $1100^{\circ}$ and $1600^{\circ} \mathrm{C}$. However, these results should be confirmed by complementary melting criteria such as the thermal metamorphism of included minerals, to avoid misinterpretation. For instance, $\mathrm{X}$-ray analysis is unable to resolve the crystal structure of nanoparticles, which tend to agglomerate in fine particulate masses showing gas segregation vesicles that are not generated by melting.

[94] The rheology of natural silicate melts is generally defined by the Navier-Stokes constitutive relationship, which postulates a linear dependence between the applied shear stress and the shear strain rate

$$
\tau=\eta \dot{\gamma}
$$

where $\eta$ is the dynamic viscosity of the melt. The viscosity of frictional melts that may form along the slip surface is likely to exert a strong influence on the dynamics of the avalanche. This parameter is mainly controlled by temperature, chemical composition, water content, and volatile and crystal content. It is probably one of the most variable and least well known rheological parameters of melts, since small variations in the previously mentioned factors may change viscosity by several orders of magnitude [Whittington et al., 2001].

[95] A simple and widely used relation for dynamic viscosity in terms of temperature and composition is given by the Arrhenius-type rheology

$$
\eta(T)=A e^{(B / T)}
$$

where $T$ is the absolute temperature $\left({ }^{\circ} \mathrm{K}\right), A$ is a reference viscosity and $B$ is an activation temperature. Nevertheless, more complex rheologies have been proposed for natural rock melts, in order to describe both the non-Newtonian behavior of lavas that contain crystals or gas bubbles [Sonder et al., 2006; Lavalle et al., 2007] and the nonArrhenian viscosity of anhydrous and hydrous silicate melts [e.g., Hui and Zhang, 2007].

[96] It is usually assumed that the presence of silicate melts along a fault is likely to reduce the shear strength to values that are much lower than those obtained for dry friction. However, high-velocity friction experiments on dry samples of gabbro suggest that the formation of a thin molten layer along a shear zone may induce a substantial increase in the shear stress up to a local peak value, presumably due to viscous effects [Fialko and Khazan, 2005; Hirose and Shimamoto, 2005]. The peak strength is followed by an exponential decrease down to a steady state value well above zero (e.g., the peak and steady state apparent friction coefficients are $\mu_{p} \sim 1$ and $\mu_{\mathrm{ss}} \sim 0.5$ ). The exponential decrease is mainly due to the reduction in the shear strain rate as the thickness of the molten layer increases toward a steady state value (e.g., $\sim 0.13 \mathrm{~mm}$ ). The peak strength and the subsequent slip weakening occur for meter to decameter slip distances, which diminish as the slip velocity increases.

[97] According to these laboratory experiments, frictional melting may act as a brake to avalanche motion. The experiments were conducted under conditions that are similar to those expected along the slip surface of a rock avalanche, excepted for the water content (slip rates of $0.85-1.5 \mathrm{~m} / \mathrm{s}$, shear strain rates of $\sim 10^{5} \mathrm{~s}^{-1}$, normal stresses of 1.2-2.4 MPa, and displacements up to $124 \mathrm{~m}$ ). Note that initial melting causes rapid strengthening of the slip surface, which restrains sliding motion and inhibits avalanche propagation. Additionally, subsequent weakening is not enough to generate thermal runaway and vanishing shear resistance, in spite of the large shear displacements.

[98] We argue that viscous forces along the slip surface of rock avalanches may be much smaller than those predicted by the laboratory experiments of Hirose and Shimamoto [2005], mainly because of the water content of rocks in the field. Water has an extremely large effect on the viscosity of molten rocks [Whittington et al., 2001]. For instance, the viscosity of melts from igneous volcanic rocks of intermediate composition may decrease more than 4 orders of magnitude, for water contents increasing from dry conditions to $5 \mathrm{wt} \%$.

[99] Recent studies have suggested that the viscosity of natural rock melts may strongly decrease as a function of shear strain rate for a wide range of temperatures [Sonder et al., 2006; Lavalle et al., 2007]. These studies, however, only explore the non-Newtonian rheology of (partially) molten rocks for shear rates $<30 \mathrm{~s}^{-1}$, whereas average shear rates along the slip surface of an avalanche are 3-4 orders of magnitude greater. Thus, new experiments are required to determine the shear rate dependence of viscosity for very high shear rates $\left(\sim 10^{5} \mathrm{~s}^{-1}\right)$ and high temperatures.

[100] The mechanism of shear melting should follow other thermal weakening processes operating at submelting temperatures. However, the occurrence of such high temperatures remains a paradox, since heat production is supposed to decrease as frictional stress diminishes. One possible explanation consists in supposing that the slip surface is not totally planar, presenting asperities at macroscopic scale. These asperities may be progressively abraded by friction. Abrasion may supply additional heat into the slip surface, contributing to the melting process. The large displacements and velocities observed in avalanches certainly enhance thermal weakening processes at submelting temperatures as well as shear melting along the slip surface.

\section{Hypothetical Example of an Avalanche Triggered by Rain}

[101] To illustrate our numerical model, we have chosen to model an avalanche mobilizing shallowly dipping layers where the stratification dips in the same direction as the hillslope. The hillslope profile and the structure are defined from the Jiufengershan rock-and-soil avalanche, which was triggered by the Chi-Chi earthquake, Taiwan, 1999 [Chang et al., 2005a].

[102] In this section, we analyze the triggering and motion of an avalanche resulting from slip along a hypothetical 


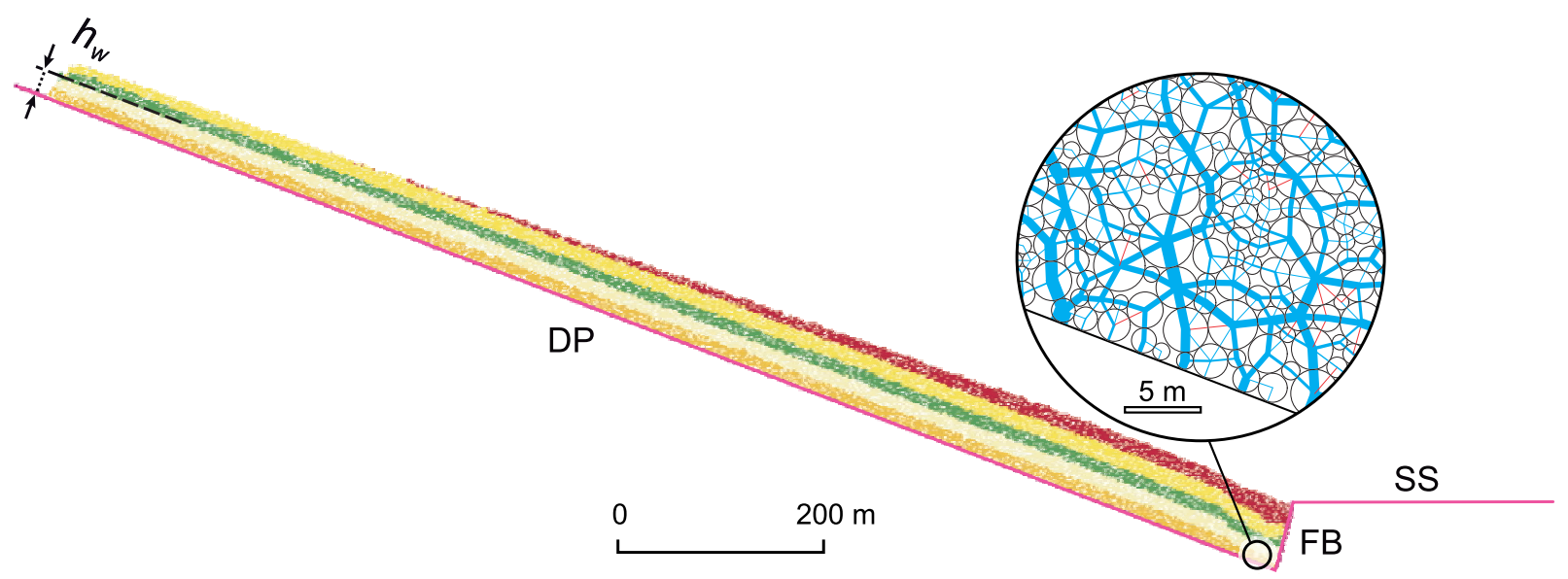

Figure 8. Hillslope granular model defining the initial geometry and the structure of the shallowly dipping layers. Close-up shows disks and initial normal contact forces between particles. Normal forces are illustrated as rectangles connecting particle centers (blue and red colors indicate compressional and extensional forces, respectively). Each rectangle width is proportional to contact force magnitude. DP, dipping plane; FB, frontal backstop; SS, surface of separation.

weak layer located at depth. The hypothetical trigger is rain, which progressively raises the water table.

\subsection{Hillslope Granular Model}

[103] The granular model was constructed from a detailed geological cross section across the Jiufengershan hillslope area [Chang et al., 2005a], and involves the geological layers destabilized during the 1999 rock-and-soil avalanche, as illustrated in Figure 8. The average thickness of the layers increases toward the footslope, from $\sim 42 \mathrm{~m}$ in the upslope area to $\sim 65 \mathrm{~m}$ downslope. To simplify the simulation, the model considers a set of 5 homogeneous layers composed of cohesive granular material. Each layer is $\sim 13.5 \mathrm{~m}$ thick and extends over $1.5 \mathrm{~km}$ along a slope dipping $21^{\circ}$ to the right. The simulation is composed of $\sim 20,000$ disks with diameters ranging between 1 and $4 \mathrm{~m}$, and the particle size distribution is uniform by volume. This particle size distribution is well adapted to simulate decametric to hectometric structures such as folds and shear planes.

[104] The contact law is assumed to be the same for the different layers (i.e., the mechanical behavior is homogeneous). The contacts between particles belonging to adjacent layers are considered as frictional and cohesionless. Thus, the bedding planes have the same frictional strength as the bulk whereas they have no tensile strength. The particle density is $2500 \mathrm{~kg} / \mathrm{m}^{3}$, which implies that the average density of the bulk is $\sim 2100 \mathrm{~kg} / \mathrm{m}^{3}$ considering a packing fraction of 0.84 . The granular layers are bounded by a set of lines named as follows (Figure 8): the dipping plane (DP), which corresponds to the potential surface of rupture of the avalanche; the frontal backstop (FB), which corresponds to the lowermost boundary of the granular model; and the surface of separation (SS).

\subsection{Shear Strength of Granular Material}

[105] We suppose that the model is composed of the weakly weathered rocks (WWR) observed in the Jiufengershan hillslope area. In Table 1 are specified the contact and the macroscopic strength parameters for the granular mate- rial simulating weakly weathered rock and for the lines that bound the granular model. The effective cohesion and the effective friction angle for the peak and the residual strength were selected from average values obtained by means of geotechnical shear tests [Chindao, 2001, 2002].

[106] The Mohr-Coulomb behavior of cohesive granular material is determined by means of biaxial numerical tests performed on a granular sample composed of 5000 particles. An example of a biaxial numerical test performed at a confining pressure of $8 \mathrm{MPa}$ is illustrated in Figure 9. The color of particles indicates rotation in radians according to the gray scale in the image (positive is counterclockwise). Shortening of the sample leads to the formation of two conjugated shear bands identified by means of particle rotations. Macroscopic shearing involves varying microscopic failure mechanisms (sliding and rolling of particles,

Table 1. Contact and Macroscopic Strength Parameters for Granular Material Simulating Weakly Weathered Rock and for the Granular Model Boundaries ${ }^{\text {a }}$

\begin{tabular}{lcccc}
\hline & WWR & DP & FB & SS \\
\hline \multicolumn{5}{c}{ Contact Parameters } \\
$\sigma_{\text {ext }}(\mathrm{kPa})$ & 100 & 100 & 100 & - \\
$\sigma_{\text {res }}(\mathrm{kPa})$ & 25 & 0 & 0 & 0 \\
$\mu_{s}$ & 0.3 & 0.3 & $0.7,0.5$ & 0.2 \\
$\mu_{r}$ & 0.1 & 0.1 & 0.1 & 0.1 \\
$\epsilon_{n}, \epsilon_{t}$ & 0.02 & 0.02 & 0.02 & 0.02 \\
\multicolumn{5}{c}{ Macroscopic Parameters } \\
$c_{\text {max }}^{\prime}(\mathrm{kPa})$ & 88 & 30 & 70 & \\
$\phi_{\text {max }}^{\prime}\left({ }^{\circ}\right)$ & 38.5 & 16.7 & 35 & - \\
$c_{\text {res }}^{\prime}(\mathrm{kPa})$ & 13 & 0 & 0 & 0 \\
$\phi_{\text {res }}^{\prime}\left({ }^{\circ}\right)$ & 26.2 & 16.7 & 26.6 & 11.3 \\
\hline
\end{tabular}

${ }^{\mathrm{a}} \mathrm{WWR}$, weakly weathered rock; DP, dipping plane; FB, frontal backstop; SS, surface of separation. Here $\sigma_{\text {ext }}$ and $\sigma_{\text {res }}$ are the maximum and residual tensile strength, $\mu_{s}$ and $\mu_{r}$ are the sliding and rolling friction coefficients (peak and residual values are given if different), $\epsilon_{n}$ and $\epsilon_{t}$ are the normalized size of the axes of the elliptic zone controlling cohesive behavior (Figure $2 \mathrm{~b}$ ), $c_{\text {max }}^{\prime}$ and $c_{\text {res }}^{\prime}$ are the maximum and residual effective cohesion, and $\phi_{\max }^{\prime}$ and $\phi_{\text {res }}^{\prime}$ are the maximum and residual effective friction angle. 


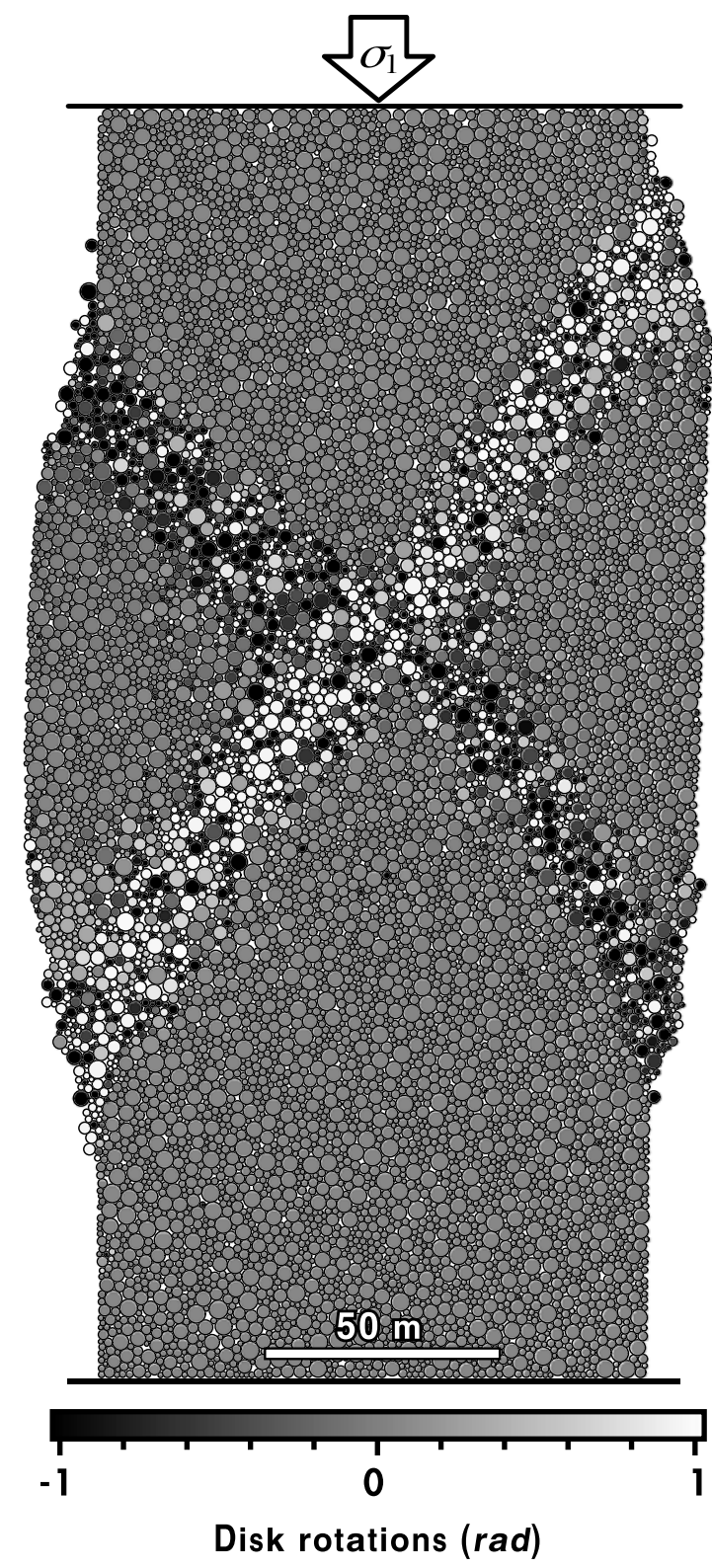

Figure 9. Granular sample subjected to a biaxial numerical test $\left(\varepsilon_{z} \approx 0.01, \sigma_{3}=8 \mathrm{MPa}\right)$. Shortening of the sample forms two conjugated shear bands. The behavior law corresponds to weakly weathered rock. Intensity of shading indicates rotation in radians according to the scale in the image (positive is counterclockwise).

and failure of strong force chains). The sense of particle rotation along shear bands follows mostly the shear direction (synthetic disks). Nevertheless, a small number of particles show antithetic rotation, as a consequence of the mechanical coupling between disks during shear. The width of shear bands is roughly 5 to 8 times the average particle diameter. The deformation pattern of the granular sample is scale independent for homothetic particle sets that share common strength properties (section 3.2.2).

[107] Figure 10 shows the stress-strain curves determined for three biaxial tests performed at different confining pressures $(4,8$, and $16 \mathrm{MPa})$. The stress-strain curves show plastic deformation with strain hardening and softening. The average maximum strength is reached for an axial strain $\varepsilon_{z}$ between 0.01 and 0.02 , while the average residual strength is observed for large axial strains $\left(\varepsilon_{z}>0.04\right)$. These values are close to the corresponding values determined in the laboratory tests in samples from the Jiufengershan hillslope area [Chindao, 2001, 2002]. The stress-strain curves show stress fluctuations resulting from shear instabilities within the samples [Taboada et al., 2005b]. Stress fluctuations reflect particle rearrangements as a distinctive feature of nonsmooth mechanical processes. The magnitude of stress fluctuations decreases with particle size, while average stresses remain constant. Note that the failure of cohesive bonds between particles is essentially controlled by average forces and not by stress fluctuations. The peak and residual strengths are determined for each curve. The Mohr-Coulomb rupture envelope for WWR is given by the line that is tangent to the Mohr circles, for five different confining pressures (Figure 11).

[108] The volumetric strain $\left(\Delta V_{s}\right)$ of the numerical samples is globally dilatant as shown in Figure 10. Dilatant behavior is characteristic of dense granular samples in which dilatant shear bands initiate and propagate. The volumetric strain increases at a high rate during the initial deformation of the sample (strain hardening and softening); it increases very slowly for large axial strains, and it is slightly higher for low confining pressures. This behavior is characteristic of dense soils and plastic deformation of rocks; however, the values of $\Delta V_{s}$ are larger than typical values for geomaterials [Goodman, 1989; Mitchell and Soga, 2005], because the ratio of particle size to sample size is much greater than in soil or rock samples. The volumetric strain shows small contraction phases which are correlated with axial stress fluctuations (see section 4.2.3). The total contraction during each of these phases is comparable with the volume of one average size particle.

\subsection{Slip Surface}

[109] In the example presented, the dipping plane (DP) and the surface of separation (SS) are considered to be weak cohesionless interfaces, whereas the frontal backstop (FB) has the same strength as the bulk (Figure 8). The macroscopic friction coefficients along the two weak interfaces are, respectively, $\mu_{\mathrm{DP}}=0.3$ and $\mu_{\mathrm{SS}}=0.2$ (see Table 1). These values are consistent with friction values observed in weak materials such as clays [e.g., Mitchell and Soga, 2005]. Note that the DP is a potential décollement level that is weaker than the weakly weathered rocks (WWR) in the bulk.

[110] We suppose that the shear strength along the surface of rupture is slip weakening, as described for many of the frictional heating mechanisms that operate along shear zones. For simplicity, we assume that the reduction in shear strength is defined by a step function (i.e., the shear strength is constant for slip distances lower than a critical slip distance $D_{c}$ and drops to zero at the critical slip distance). This approximation mimics the sharp (e.g., exponential) decrease in shear strength as a function of slip distance, determined from laboratory experiments and numerical simulations (see section 4.3). The value of $D_{c}$ ranges between a few centimeters and several meters depending on the weakening mechanism and on parameters such as the confining pressure, the permeability of fault gouge, and the 


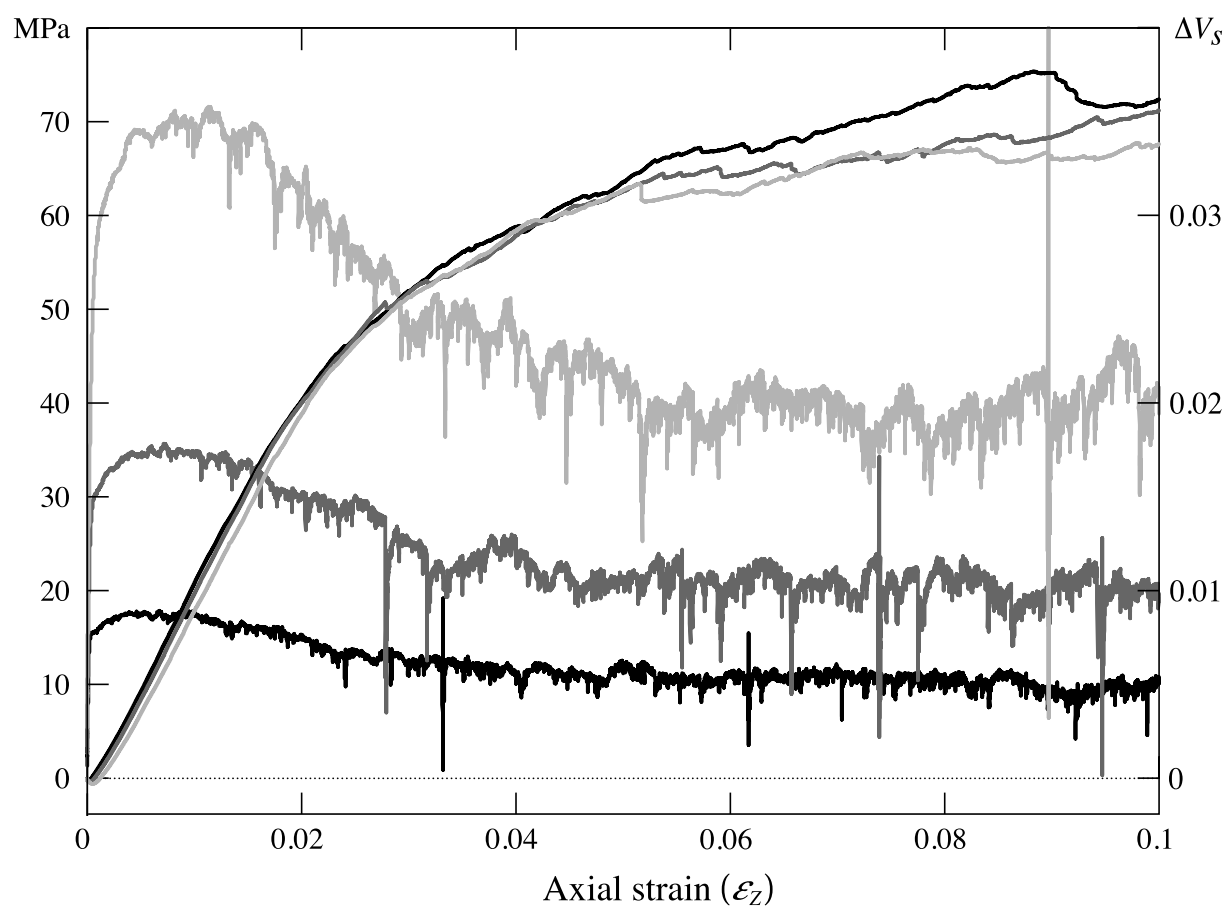

Figure 10. Stress-strain curves and volumetric strains for the granular sample in Figure 9, determined from biaxial tests performed at three confining pressures (black is 4 , dark gray is 8 , and light gray is $16 \mathrm{MPa}$ ). Stress-strain curves show a well-marked peak followed by a residual strength plateau. Volumetric strain curves show dilatant behavior.

thickness of the shear zone. We have set $D_{c}=5 \mathrm{~m}$, which is consistent with weakening slip distances for the models of rock avalanches with supposed thermal pressurization of the pore fluid [e.g., Chang et al., 2005b; Veveakis et al., 2007]. Nevertheless, the dynamic behavior of the granular hillslope has been analyzed for other threshold distances $(1-10 \mathrm{~m})$; this parameter only affects the response time of avalanche triggering and does not modify the main results of this study.

\subsection{Pore Water Pressure}

[111] In our hypothetical example, we suppose that the water table is located at a constant height above the potential surface of rupture (Figure 4). This approximation is frequently used in infinite slope stability analyses, in which the surface of seepage is parallel to the slope [e.g., Duncan, 1996]. In this situation, the pressure gradient can be accurately estimated by

$$
\nabla p=\gamma_{w} \cos \beta,
$$

where $\gamma_{w}=9.81 \mathrm{kN} / \mathrm{m}^{3}$ is the unit weight of water and $\beta$ is the dip of the layers. The volume force $F_{p}$, which simulates the effect of pore pressure on a particle, is perpendicular to the slope and points upward (Figure 4). Thus, the pore water pressure reduces the normal component of the particle weight, decreasing the normal effective stress along the DP.

[112] In our conceptual model, we suppose that pore water pressure in the bulk is negligible once the avalanche is triggered and dilatant deformation initiates. This condition is introduced in the numerical approach by deactivating the volume force $F_{p}$, once the particle has moved down- slope a distance greater than the critical distance $D_{c}$. This criterion is applied to all the particles located beneath the water table. Note that the same critical distance is used to vanish frictional strength along the surface of rupture and to release pore water pressure at particle scale. Thus, the pore pressure release occurs simultaneously with slip weakening and avalanche triggering.

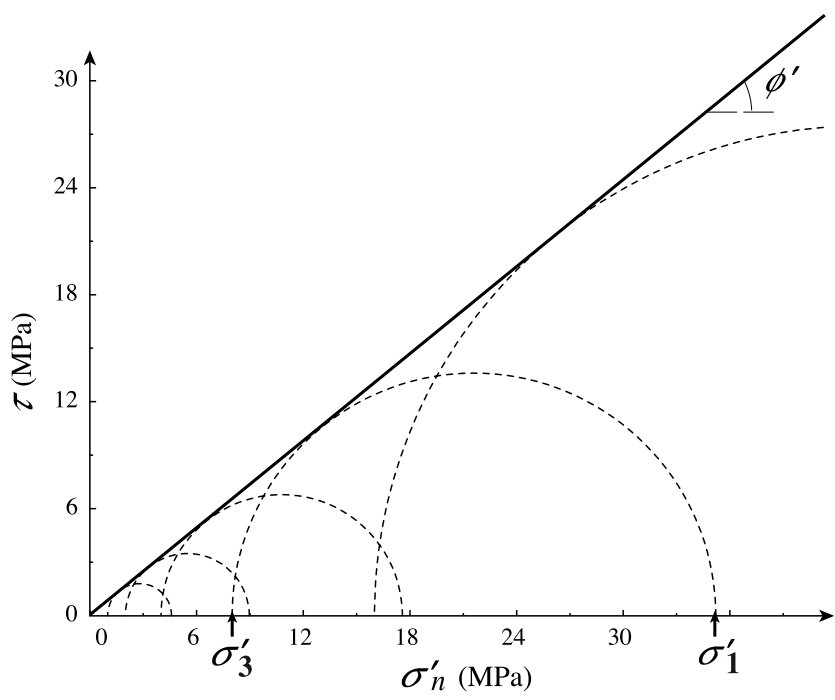

Figure 11. Peak failure envelope for the sample in Figure 9, obtained from five biaxial numerical tests performed at different confining pressures. Here $\sigma_{n}^{\prime}$ is the effective normal stress, $\tau$ is the shear stress, and $\phi^{\prime}$ is the peak effective friction angle. 

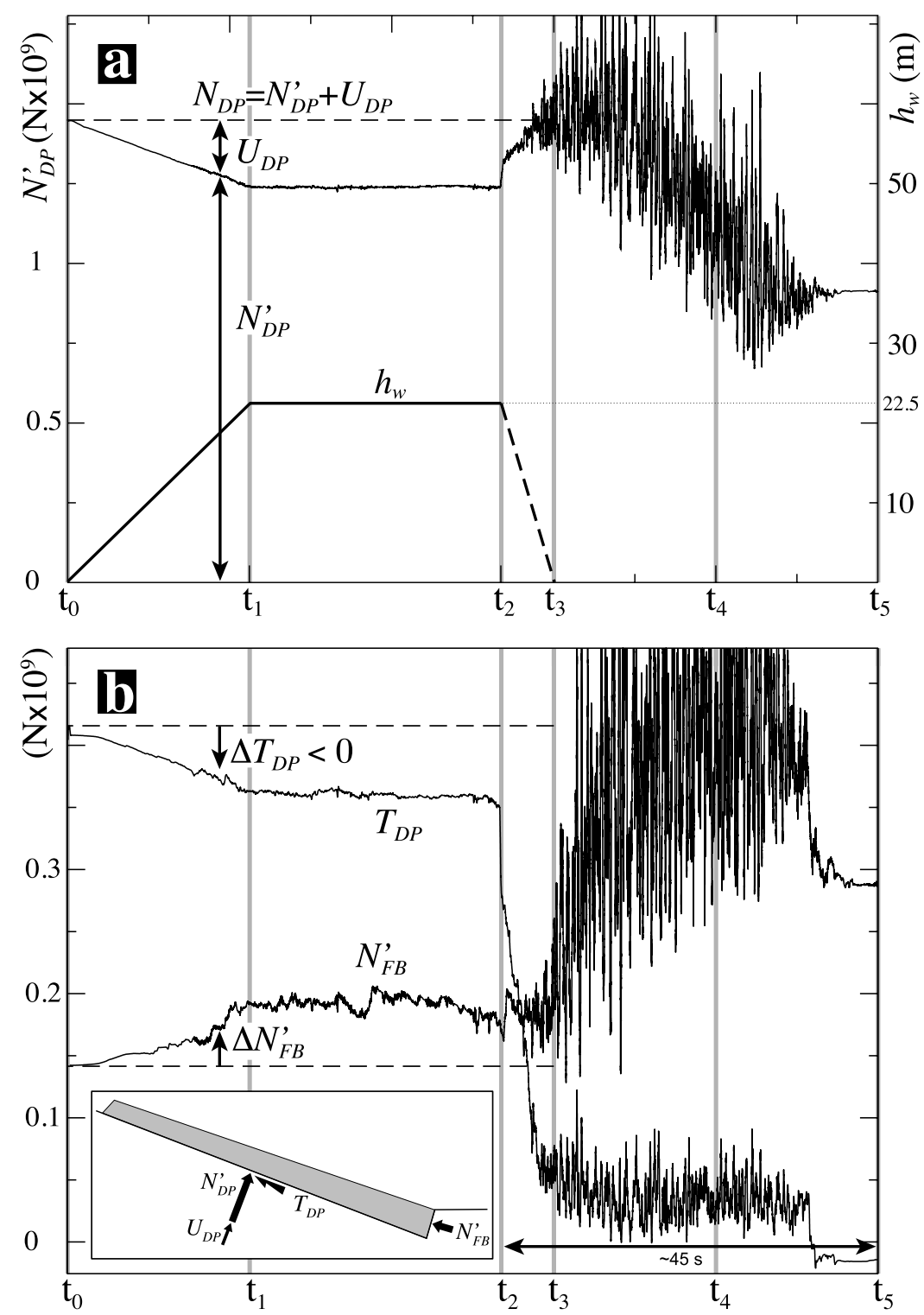

Figure 12. Normal and shear forces applied to the granular system along the DP and the FB as a function of time (see inset). (a) Total normal force $N_{D P}$, normal effective force $N_{D P}^{\prime}$, force applied by the water pressure $U_{D P}$, and water table height $h_{w}$; (b) shear force $T_{D P}$, normal effective force $N_{F B}^{\prime}$, and variations in the shear and normal effective forces $\Delta T_{D P}$ and $\Delta N_{F B}^{\prime}$. Times $t_{0}, . ., t_{5}$ are characteristic time values in avalanche process (see text for explanations).

[113] More realistic criteria for pore pressure release could be introduced. For instance, the pore pressure release may be a function of the local dilatancy in the vicinity of individual particles. A local dilatancy criterion is likely to delay the pore pressure release, therefore decreasing the frictional strength of cohesive bonds during incipient deformation. Nevertheless, the specific criterion implemented for releasing pore pressure does not have a major influence on the final deformation of the hillslope, provided that the avalanche is triggered before pore pressure release.

\section{Simulation Results}

[114] To simulate an avalanche triggered by rain we set up a simple numerical experiment consisting of progressively raising the height of the water table until the tilted layers became unstable. In the initial state, the water table is located along the dipping plane, and it remains parallel to the granular layers as it rises. Figure 12 shows the forces exerted on the dipping plane (DP) and on the frontal backstop (FB) as a function of time. The shear and normal effective forces that are applied on the particle set along these planes are named, respectively, $T_{\mathrm{DP}}, N_{\mathrm{DP}}^{\prime}$ and $N_{\mathrm{FB}}^{\prime}$, as indicated in the inset. Four different stages are observed in the mechanical behavior of the granular system. These stages are described in detail in the following sections in terms of the magnitude of forces at the boundaries and the kinematical configuration of the granular layers (Figures 12 and 13). Animation S1 is also available as auxiliary material. ${ }^{1}$

${ }^{1}$ Auxiliary materials are available in the HTML. doi:10.1029/ 2008JF001072. 


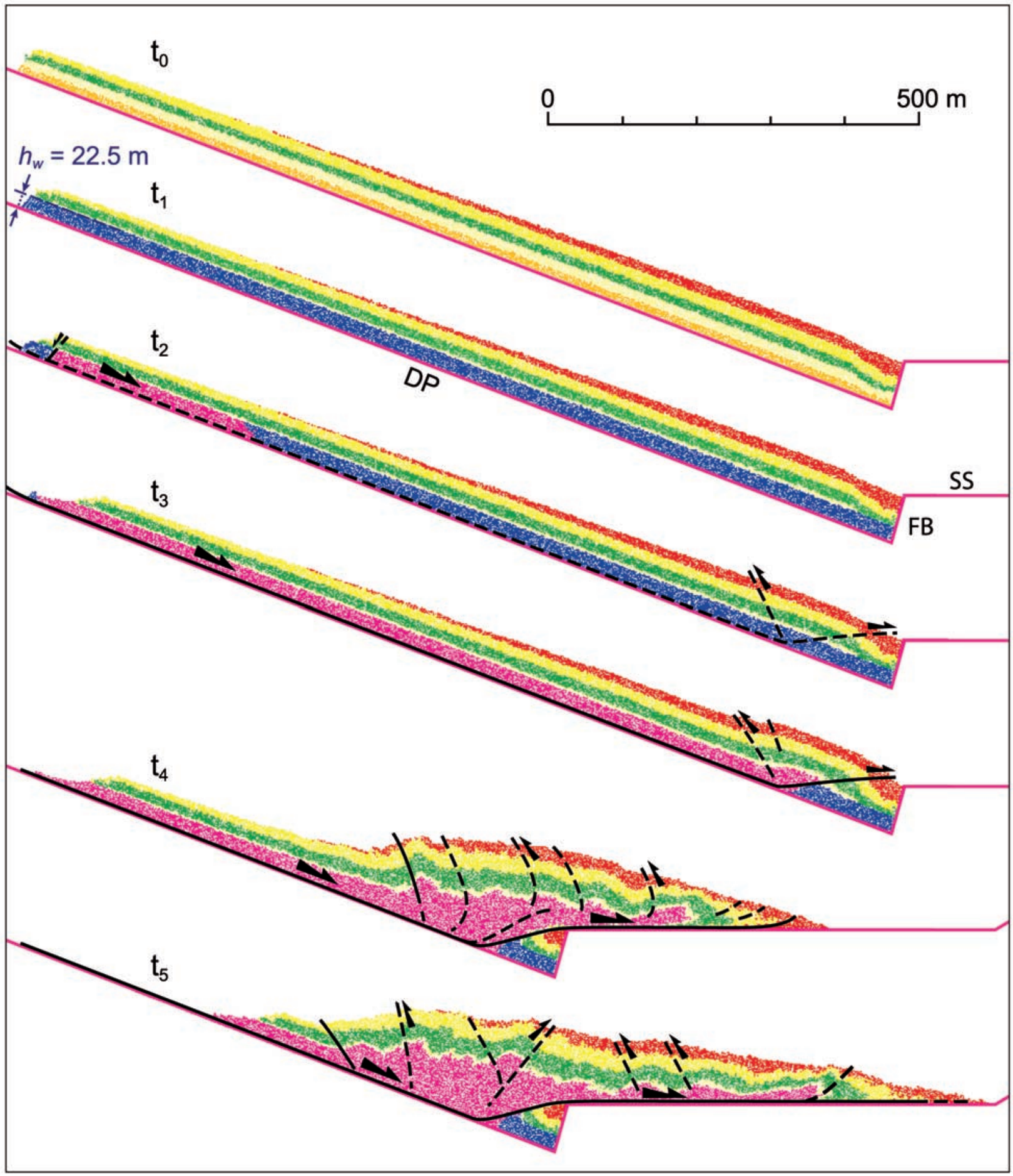

Figure 13. Snapshots illustrating the kinematic state of the hillslope at characteristic times of avalanche triggering and motion (see Figure 12 and text). Blue areas are subjected to pore water pressure in the bulk, while the pink areas indicate pore pressure release and slip weakening along the DP.

\subsection{Water Table Rise}

[115] In the interval $\left[t_{0}, t_{1}\right]$ the water table height $h_{w}$ rises linearly with time from 0 to $22.5 \mathrm{~m}$. Particles located beneath the water table are indicated in blue (Figure 13, $\left.t_{1}\right)$. During this stage, the behavior of the granular layers is quasistatic and only small rearrangements in the particle system are observed. In natural conditions, the rate at which the water table rises is variable $(0.01-1 \mathrm{~m} / \mathrm{h})$, and it depends on the precipitation events and on the infiltration capacity. However, the duration of this phase is generally very long compared to the duration of the failure and triggering phases. Equilibrium conditions require that the total normal force $N_{\mathrm{DP}}$ applied at the potential surface of rupture is constant. $N_{\mathrm{DP}}$ can be decomposed into two 
distinct terms (Figure 12a): the effective normal force $N_{\mathrm{DP}}^{\prime}$ and the force $U_{\mathrm{DP}}$ resulting from pore water pressure. The rise in the water table increases the pore water pressure at the DP, reducing the effective normal force $N_{\mathrm{DP}}^{\prime}$. The shear force $T_{\mathrm{DP}}$ decreases linearly during this interval (Figure 12b). The variation in shear force can be approximated by

$$
\Delta T_{\mathrm{DP}} \approx \mu_{\mathrm{DP}} \Delta N_{\mathrm{DP}}^{\prime} \approx-\mu_{\mathrm{DP}} U_{\mathrm{DP}}
$$

where $\mu_{\mathrm{DP}}$ is the coefficient of sliding friction at the DP, and $\Delta T_{\mathrm{DP}}$ and $\Delta N_{\mathrm{DP}}^{\prime}$ are the variations in the shear and normal effective forces applied along the DP. This relation indicates that the applied shear stress is at the threshold value given by

$$
\tau_{\max }=\mu_{\mathrm{DP}} \sigma_{n}^{\prime}
$$

where $\sigma_{n}^{\prime}$ is the normal effective stress. This behavior is not surprising since the slope of the dipping plane is greater than its friction angle $\left(21^{\circ}>16.7^{\circ}\right.$, see Table 1). During this stage, the overall stability of the tilted layers is assured by the normal force $N_{\mathrm{FB}}^{\prime}$ applied at the frontal backstop, which increases linearly and in the same amount as the decrease in shear force

$$
\Delta N_{\mathrm{FB}}^{\prime} \approx-\Delta T_{\mathrm{DP}}
$$

\subsection{Quasistatic Failure of the Hillslope}

[116] In the interval $\left[t_{1}, t_{2}\right]$ the water table height $h_{w}$ reaches a critical value at which shear rupture of the granular layers is initiated simultaneously along the dipping plane and within the footslope area. At time $t_{1}$ the normal force $N_{\mathrm{FB}}^{\prime}$ reaches the compressional strength of the granular layers and it remains fairly constant during the time interval. At this stage the limit equilibrium of the granular layers is achieved, failure is incipient, and the landslide may be considered as an incipient rockslide. Two conjugated shear bands are initiated within the footslope area where compressional stresses parallel to the hillslope are highest (Figure 13, $t_{2}$ ). Macroscopic plastic deformation concentrates around the shear bands and along the surface of rupture DP.

\subsection{Avalanche Triggering}

[117] Avalanche triggering occurs during the interval $\left[t_{2}\right.$, $\left.t_{3}\right]$, when the tilted layers accelerate downslope, passing rapidly from quasistatic to dynamic conditions ( $\sim 5 \mathrm{~s})$. During this phase, the shear resistance $T_{\mathrm{DP}}$ at the surface of rupture drops drastically. The reduction in shear resistance is linked with slip weakening as particle slip surpasses the critical distance (see section 5). The area where pore pressure is released is apparent as the color of particles changes from blue to pink (Figure 13). In this zone, the drop in shear resistance operates along the surface of rupture: the frictionless area propagates from the upper slope area toward the footslope. As the shear strength drops to zero, the granular layers accelerate downslope since the gravitational force component parallel to the basal plane is uncompensated. The normal force $N_{\text {DP }}^{\prime}$ increases slightly as the external forces representing pore water pressure deactivate.

\subsection{Avalanche Motion}

[118] Avalanche motion takes place in the time interval $\left[t_{3}, t_{5}\right]$ and lasts roughly $35 \mathrm{~s}$. The intermediate and final state of the granular layers during avalanche motion are illustrated in Figure 13 (times $t_{4}$ and $t_{5}$ ). Granular layers are progressively deformed and folded as they reach the downslope area and override the surface of separation. The contact forces at the boundaries show large fluctuations at small timescales from particle collisions and intense deformation of the granular layers. These fluctuations are probably not representative of stress fluctuations during a real rock avalanche, in which particles are, on average, much smaller. However, the average values of forces along boundaries should be similar to those observed in the model, showing the following trends (Figure 12): (1) The normal force $N_{\text {DP }}^{\prime}$ decreases as the granular layers move downslope. (2) The normal force $N_{\mathrm{FB}}^{\prime}$ increases with the momentum of the granular layers located on the DP. (3) The shear force $T_{\mathrm{DP}}$ oscillates around a small positive value.

[119] The normal force $N_{\mathrm{FB}}^{\prime}$ stabilizes at a higher value once the avalanche comes to rest; this result is consistent with the drop in shear strength along the DP during the avalanche and thickening of the granular deposit. Note that the lowermost portion of the DP is located beneath the slip surface, and therefore has a nonweakened shear strength. Thus, the shear force $T_{\mathrm{DP}}$ along this portion, shows low positive and negative values during and after avalanche motion, respectively. Negative values indicate that the shear sense at the footslope is inverted during postavalanche relaxation.

\section{Summary and Discussion}

[120] We present a discrete element simulation of a granular avalanche that integrates initiation and motion phases. The model is based on Contact Dynamics and integrates the main components that control landslides, which are as follows: hillslope and slip surface geometry; rock materials (modeled as cohesive granular material); and trigger (e.g., intense rain, seismic ground acceleration, or toe erosion).

[121] Prior to avalanche triggering (i.e., in quasistatic conditions), the behavior of granular material is defined in terms of a Mohr-Coulomb law. The internal friction angle and the cohesion depend on the strength parameters at contact scale (sliding and rolling friction coefficients and adhesion). The contact-scale parameters of the granular material corresponding to a macroscopic strength are determined through numerical biaxial tests. The effect of groundwater on the stability of the hillslope is introduced in the model by applying pore water pressure forces to particles.

[122] During avalanche motion (i.e., in dynamic conditions), pore water pressure in the bulk is neglected, and, accordingly, the avalanche is modeled as a dense granular flow of dry, frictional and cohesive particles.

[123] Rock avalanches are typically characterized by an unexpectedly long-runout distance, whose origin is still a matter of debate. We have reviewed some of the main theories that try to explain the mechanical origin of long runout in the light of granular physics. According to our analysis, long runout appears to have many causes, which are linked to the type of rock avalanche. From a mechanical 
point of view, the following two types of rock avalanches are to be distinguished: (1) those for which the slip surface is stronger than the bulk (e.g., the slip surface is rough), in which case the shear strain will be mostly distributed within the bulk and (2) those for which the slip surface is weaker than the bulk (e.g., the slip surface is smooth), in which case the shear displacement will tend to concentrate along the slip surface.

[124] Some of the theories that try to explain the long runout of rock avalanches mobilized over rough interfaces, are inconsistent with the mechanical behavior of granular materials. Two of these theories advance hypotheses stating that agitation of granular material may reduce the frictional strength and therefore increase the runout distance. Firstly, the mechanical fluidization theory proposes that the bulk rides over a thin layer of rapidly sheared particles whose frictional strength is lowered as a consequence of agitation. Secondly, the acoustic fluidization theory proposes that the random vibrations of groups of particles, generated during the collapse of a mass of rock, may relieve overburden stresses and reduce the frictional resistance between clasts. Conversely, many studies, experimental and numerical, show that sheared granular materials are strain rate strengthening, and indicate that, as the shear strain rate increases, granular material becomes more agitated, and hence more dissipative and more resistant. A third theory, invokes that a rock avalanche may be imaged as a biphased granular material consisting of a dense granular flow and an interstitial fluidized phase (i.e., a granular gas) generated by dynamic fragmentation of clasts. The granular gas creates an isotropic pressure that reduces normal contact forces in the force network of the dense granular flow, driving longer runout. Our interpretation of granular mechanics suggests that fragmentation may locally induce transient fluidization and strengthening of the granular material, slowing down the granular flow. Contrastingly, the reduction of particle size and the decrease in average axial stresses along strong force chains, both resulting from fragmentation, are likely to weaken the granular material, enhancing long runout.

[125] According to some simple numerical and experimental models of granular flows, the long runout of an avalanche that propagates over a rough slip surface may be controlled by the initial geometry of the rock mass rather than by the details of the interactions between rock fragments. In this setting, long runout is enhanced for columnlike rock masses that collapse from steep hillslopes and that show a large aspect ratio at the initial state (i.e., the ratio between height and width). Motion of a granular avalanche over a rough horizontal plane may show three flow regimes with differing velocity profiles: A plug flow near the front of the avalanche, a homogeneous simple shear flow located upstream, and a two-layered flow at the rear of the avalanche, composed of a shearing layer overlying a static layer. In this flow configuration, shear strain migrates progressively from the lowermost part of the granular flow toward the top. The succession between the three flow regimes is closely related to the dissipation of kinetic energy in the granular flow. The total shear strain is homogeneously distributed within the entire granular mass, favoring long runout.
[126] Long runout may also be linked to dynamic weakening mechanisms that affect the slip surface, many of which have been postulated as agents that weaken seismic faults during earthquakes. Among these mechanisms, we suggest that those involving thermal weakening may be relevant to the dynamics of rock avalanches. The more significant thermal weakening mechanisms are thermal pressurization of pore fluid, degradation of microcontacts by flash heating, thermal decomposition of rock minerals, lubrication by silica gel, and shear melting. All these processes may lower drastically the shear strength along the slip surface, from avalanche initiation onward. We advance the hypothesis that, with few rare exceptions, a large rockslide may transform into an avalanche provided that the slip surface has the following two distinctive features: (1) The slip surface, or at least the surface of rupture, should exhibit dynamic weakening during triggering and motion of the landslide. (2) The geometry of the slip surface should be curvilinear, or alternatively, the surface of separation should be rough. Dynamic weakening of the slip surface may induce mobilization of the bulk at high velocity; sliding along a curvilinear slip surface may induce deformation and comminution of the bulk, even if sliding friction is very low; and sliding along a rough surface of separation may induce heterogeneous shear flow and comminution of the bulk.

[127] The model is illustrated with a hypothetical example of a rain-triggered avalanche, which mobilizes shallowly dipping layers of weakly weathered rock. The numerical experiment progressively raises the water table until the dipping layers become unstable. The avalanche trigger is a simple slip-weakening criterion along the surface of rupture. Several phases in the avalanche are identified: an initial static phase characterized by pore water pressure increase as a result of the water table rise, a quasistatic failure of the hillslope at a critical water height (i.e., the rockslide stage), avalanche triggering from the drop in shear strength along the surface of rupture as a consequence of slip weakening, and an extremely dynamic phase of avalanche motion in which rocks are intensely folded and sheared.

[128] Several improvements and extensions can be introduced in the numerical approach in order to achieve a more realistic simulation of dynamic surface processes. For example, the avalanche triggering phase may consider the thermomechanical properties of the slip surface. These properties may be correlated with physical parameters in the granular system such as the frictional heat production, the slip, and the slip rate at line-disk contacts. The frictional heat production can be calculated from the energy dissipated by shear forces at line-disk slipping contacts.

[129] This simulation methodology is well adapted to investigate the morphological evolution of hillslopes subjected to bedrock landslides and avalanches, taking into account tectonic and climatic forcing as well as complex boundary conditions such as erosion and weathering of surface materials. This approach can be coupled with hillslope evolution models based on slope-dependent transport laws [Densmore et al., 1998; Roering et al., 2001]. With increasing computation capacity, the simulation technique can be extended to analyze three-dimensional landslides involving a much greater number of particles. In principle, 
geometrical similarity between model and a rock avalanche can only be achieved by using 3-D particles.

[130] Acknowledgments. We thank K.-J. Chang and F. Radjaï for discussions on granular mechanics and participation in the development of simulation programs and A. Delplanque for technical help. We thank M. McSaveney for a thorough review of the manuscript and for insightful discussions on avalanche mechanics. This work was partially funded by the following projects: INSU Reliefs de la Terre 2005-2006, ANR ACTSTaiwan 2006-2010, and Ecos-Nord C08U01 2008-2010.

\section{References}

Azéma, E., F. Radjaï, R. Peyroux, and G. Saussine (2007), Force transmission in a packing of pentagonal particles, Phys. Rev. E, 76, $011301-$ 011314, doi:10.1103/PhysRevE.76.011301.

Bear, J. (1988), Dynamics of Fluids in Porous Media, Dover, New York. Bizzarri, A., and M. Cocco (2006), A thermal pressurization model for the spontaneous dynamic rupture propagation on a three-dimensional fault: 1 . Methodological approach, J. Geophys. Res., 111, B05303, doi:10.1029/ 2005JB003862.

Buss, E., and A. Heim (1881), Der Bergsturz von Elm, 113 pp., Worster, Zurich, Switzerland

Campbell, C. S. (1989), Self-lubrication for long runout landslides, J. Geol., 97, 653-665.

Campbell, C. S. (2006), Granular material flows-An overview, Powder Technol., 162, 208-229, doi:10.1016/j.powtec.2005.12.008.

Campbell, C. S., P. Cleary, and M. Hopkins (1995), Large-scale landslide simulations: Global deformation, velocities, and basal friction, J. Geophys. Res., 100(B5), 8267-8283.

Carmona, H. A., F. K. Wittel, F. Kun, and H. J. Herrmann (2008), Fragmentation processes in impact of spheres, Phys. Rev. E, 77, 051302051312, doi:10.1103/PhysRevE.77.051302.

Chang, K.-J., A. Taboada, and Y.-C. Chan (2005a), Geological and morphological study of the jiufengershan landslide triggered by the Chi-Chi Taiwan earthquake, Geomorphology, 71(3-4), 293-309, doi:10.1016/ j.geomorph.2005.02.004.

Chang, K.-J., A. Taboada, M.-L. Lin, and R.-F. Chen (2005b), Analysis of landsliding by earthquake shaking using a block-on-slope thermomechanical model: Example of Jiufengershan landslide, central Taiwan, Eng. Geol., 80(1-2), 151-163, doi:10.1016/j.enggeo.2005.04.004.

Chevoir, F., M. Prochno, J. T. Jenkins, and P. Mills (2001), Dense granular flows down and inclined plane, in Powders and Grains 2001, edited by Y. Kishino, pp. 373-376, A. A. Balkema, Brookfield, Vermont.

Chindao (2001), Borehole investigations in the Jiufengershan landslide-1 (in Chinese), technical report, Chindao Eng. Consult. Ltd. and Natl. Taiwan Univ., Taipei.

Chindao (2002), Borehole investigations in the Jiufengershan landslide-2 (in Chinese), technical report, Chindao Eng. Consult. Ltd. and Natl. Taiwan Univ., Taipei.

Collins, G. S., and H. J. Melosh (2003), Acoustic fluidization and the extraordinary mobility of sturzstorms, J. Geophys. Res., 108(B10), 2473, doi:10.1029/2003JB002465.

Cruden, D. M., and D. J. Varnes (1996), Landslide types and processes, in Landslides: Investigation and Mitigation, edited by A. K. Turner and R. L. Schuster, chapter 3, pp. 36-75, Natl. Acad. Press, Washington, D. C.

Cundall, P. A., and O. D. L. Stack (1979), A discrete numerical model for granular assemblies, Géotechnique, 29(1), 47-65.

Da Cruz, F., F. Chevoir, J.-N. Roux, and I. Iordanoff (2004), Macroscopic friction of dry granular materials, in Transient Processes in Tribology: Proceedings of the 30th Leeds-Lyon Symposium on Tribology, edited by G. D. A. Lubrecht, 43, pp. 53-62, Elsevier, Amsterdam.

Daudon, D., J. Lanier, and M. Jean (1997), A micromechanical comparison between experimental results and numerical simulation of a biaxial test on 2-D granular material, in Powders and Grains 1997, edited by R. P. Behringer and J. T. Jenkins, pp. 219-222, A. A. Balkema, Brookfield, Vermont.

Davies, T. R. H. (1982), Spreading of rock avalanche debris by mechanical fluidization, Rock Mechanics, 15, 9-24.

Densmore, A. L., R. S. Anderson, B. G. McAdoo, and M. A. Ellis (1997), Hillslope evolution by bedrock landslides, Science, 275(5298), 369-372, doi:10.1126/science.275.5298.369.

Densmore, A. L., M. A. Ellis, and R. S. Anderson (1998), Landsliding and the evolution of normal fault-bounded mountains, J. Geophys. Res., 103(B7), 15,203-15,219, doi:10.1029/98JB00510.

Di Toro, G., D. L. Goldsby, and T. E. Tullis (2004), Friction falls towards zero in quartz rock as slip velocity approaches seismic rates, Nature, 427, $436-460$.
Douady, S., B. Andreotti, A. Daerr, and P. Clad (2002), From a grain to avalanches: On the physics of granular surface flows, C. R. Phys., 3(2), 177-186, doi:10.1016/S1631-0705(02)01310-5.

Duncan, J. M. (1996), Soil slope stability analysis, in Landslides: Investigation and Mitigation, edited by A. K. Turner and R. L. Schuster, chapter 13, pp. 337-371, Natl. Acad. Press, Washington, D. C.

Erismann, T. H. (1979), The mechanics of large landslides, Rock Mech Rock Eng., 12, 5-46, doi:10.1007/BF01241087.

Estrada, N., A. Taboada, and F. Radjaï (2008), Shear strength and force transmission in granular media with rolling resistance, Phys. Rev. E, 78, 021301-021312, doi:10.1103/PhysRevE.78.021301.

Fialko, Y., and Y. Khazan (2005), Fusion by earthquake fault friction: Stick or slip?, J. Geophys. Res., 110, B12407, doi:10.1029/2005JB003869.

Forterre, Y., and O. Pouliquen (2008), Flows of dense granular media, Annu. Rev. Fluid Mech., 40, 1-24, doi:10.1146/annurev.fluid.40. 111406.102142.

Friedmann, S. J., N. Taberlet, and W. Losert (2006), Rock-avalanche dynamics: Insights from granular physics experiments, Int. J. Earth Sci., 95 , 911-919, doi:10.1007/s00531-006-0067-9.

Goodman, R. E. (1989), Introduction to Rock Mechanics, 2nd ed., John Willey, New York.

Groupement de Recherche Milieux Divises (2004), On dense granular flows, Eur. Phys. J. E, 14, 341-365, doi:10.1140/epje/i2003-10153-0.

Han, R., T. Shimamoto, T. Hirose, J.-H. Ree, and J. Ando (2007), Ultralow friction of carbonate faults caused by thermal decomposition, Science, 316(5826), 878-881, doi:10.1126/science.1139763.

Heim, A. (1932), Bergsturz und Menschenleben, 218 pp., Fretz und Wasmuth, Zurich, Switzerland.

Hewitt, K. (2009), Rock avalanches that travel onto glaciers and related developments, Karakoram Himalaya, inner Asia, Geomorphology, 103(1), 66-79, doi:10.1016/j.geomorph.2007.10.017.

Hirose, T., and T. Shimamoto (2005), Growth of a molten zone as a mechanism of slip weakening of simulated faults in gabbro during frictional melting, J. Geophys. Res., 110, B05202, doi:10.1029/2004JB003207.

Hui, H., and Y. Zhang (2007), Toward a general viscosity equation for natural anhydrous and hydrous silicate melts, Geochim. Cosmochim. Acta, 71, 403-416, doi:10.1016/j.gca.2006.09.003.

Hungr, O. (2006), Rock avalanche occurrence, process and modelling, in Landslides From Massive Rock Slope Failure, Earth Environ. Sci. Ser., vol. 302, edited by S. G. Evans et al., pp. 243-266, Springer, Dordrecht, Netherlands.

Hungr, O., and N. R. Morgenstern (1984), Experiments in high velocity open channel flow of granular materials, Géotechnique, 34, 405-413.

Iordanoff, I., and M. M. Khonsari (2004), Granular lubrication: Toward an understanding of the transition between kinetic and quasi-fluid regime, J. Tribol., 126(1), 137-145.

Jean, M. (1995), Frictional contact in collections of rigid or deformable bodies: Numerical simulation of geomaterials, in Mechanics of Geometrical Interfaces, edited by A. P. S. Selvadurai and M. J. Boulon, pp. 463-486, Elsevier, New York.

Jean, M. (1999), The non smooth Contact Dynamics method, Comput. Methods Appl. Mech. Eng., 177(3-4), 235-257, doi:10.1016/S00457825(98)00383-1.

Lachenbruch, A. H. (1980), Frictional heating, fluid pressure, and the resistance to fault motion, J. Geophys. Res., 85, 6097-6122.

Lajeunesse, E., J. B. Monnier, and G. M. Homsy (2005), Granular slumping on a horizontal surface, Phys. Fluids, 17, 103302-103315, doi:10.1063/ 1.2087687.

Lavalle, Y., K.-U. Hess, B. Cordonnier, and D. B. Dingwell (2007), NonNewtonian rheological law for highly crystalline dome lavas, Geology, 35(9), 843-846, doi:10.1130/G23594A.1.

Legros, F. (2002), The mobility of long-runout landslides, Eng. Geol., 63(3-4), 301-331, doi:10.1016/S0013-7952(01)00090-4.

Lin, A., A. Chen, C. F. Liau, C. T. Lee, C. C. Lin, P. S. Lin, S. C. Wen, and T. Ouchi (2001), Frictional fusion due to co-seismic landsliding during the 1999 Chi-Chi (Taiwan) $M_{L} 7.3$ earthquake, Geophys. Res. Lett., 28, $4011-4014$

Marketos, G., and M. D. Bolton (2005), Compaction bands as observed in DEM simulations, in Powders and Grains: 5th International Conference on Micromechanics of Granular Media, Stuttgart, vol. 2, edited by R. García-Rojo, H. J. Herrmann, and S. McNamara, pp. 1405-1408, A. A. Balkema, Brookfield, Vermont.

Marone, C. (1998), Laboratory-derived friction laws and their application to seismic faulting, Annu. Rev. Earth Planet. Sci., 26, 643-696.

Masch, L., H. R. Wenk, and E. Preuss (1985), Electron microscopy study of hyalomylonites-Evidence for frictional melting in landslides, Tectonophysics, $115(1-2), 131-160$.

McSaveney, M. J., and T. R. H. Davies (2006), Rapid rock mass flow with dynamic fragmentation: Inferences from the morphology and internal 
structure of rockslides and rock avalanches, in Landslides From Massive Rock Slope Failure, Earth Environ. Sci. Ser., vol. 302, edited by S. G. Evans et al., pp. 243-266, Springer, Dordrecht, Netherlands.

Mitchell, J. K., and K. Soga (2005), Fundamentals of Soil Behavior, 3rd ed. John Willey, Upper Saddle River, N. J.

Moreau, J. J. (1988), Unilateral contact and dry friction in finite freedom dynamics, in Nonsmooth Mechanics and Applications, vol. 302, edited by J. J. Moreau and P. D. Panagiotopoulos, pp. 1-82, Springer, New York. Moreau, J. J. (1993), New computation methods in granular dynamics, in Powders and Grains 1993, edited by C. Thornton, pp. 227-232, A. A. Balkema, Brookfield, Vermont

Moreau, J. J. (1994), Some numerical methods in multibody dynamics: Application to granular materials, Eur. J. Mech. A, 13(4), 93-114.

Moreau, J. J. (1999), Some basics of unilateral dynamics, in Unilateral Multibody Contacts, edited by F. Pfeiffer and C. Glocker, pp. 1-14, Springer, New York.

Mourgues, R., and P. R. Cobbold (2003), Some tectonic consequences of fluid overpressures and seepage forces as demonstrated by sandbox modelling, Tectonophysics, 376, 75-97, doi:10.1016/S0040-1951(03)00348-2

Perez, J. A., S. B. Kachuck, and G. A. Voth (2008), Visualization of collisional substructure in granular shock waves, Phys. Rev. E, 78, 041309041315, doi:10.1103/PhysRevE.78.041309.

Pollet, N., and J. L. Schneider (2004), Dynamic disintegration processes accompanying transport of the Holocene Flims sturzstrom (Swiss Alps), Earth Planet. Sci. Lett., 221, 433-448, doi:10.1016/S0012821X(04)00071-8.

Pöschel, T., and T. Schwager (2005), Computational Granular Dynamics: Models and Algorithms, Springer, Berlin.

Pouliquen, O., C. Cassar, Y. Forterre, P. Jop, and M. Nicolas (2005), How do grains flow: Towards a simple rheology for dense granular flows, in Powders and Grains: 5th International Conference on Micromechanics of Granular Media, Stuttgart, vol. 2, edited by R. García-Rojo, H. J. Herrmann, and S. McNamara, pp. 859-865, A. A. Balkema, Brookfield, Vermont.

Radjaï, F., M. Jean, J. J. Moreau, and S. Roux (1996), Force distributions in dense two-dimensional granular systems, Phys. Rev. Lett., 77(2), 274277, doi:10.1103/PhysRevLett.77.274.

Rempel, A. W., and J. R. Rice (2006), Thermal pressurization and onset of melting in fault zones, J. Geophys. Res., 111, B09314, doi:10.1029/ 2006JB004314.

Rice, J. R. (1999), Flash heating at asperity contacts and rate-dependent friction, Eos Trans. $A G U, 80(46)$, Fall Meet. Suppl., Abstract F6811.

Rice, J. R. (2006), Heating and weakening of faults during earthquake slip, J. Geophys. Res., 111, B05311, doi:10.1029/2005JB004006.

Roering, J. J., J. W. Kirchner, and W. E. Dietrich (2001), Hillslope evolution by nonlinear slope-dependent transport: Steady-state morphology and equilibrium adjustment timescales, J. Geophys. Res., 106(B8), 16,499-16,513, doi:10.1029/2001JB000323.

Rognon, P. G., J.-N. Roux, and M. Naaim (2007), Dense flows of bidisperse assemblies of disks down an inclined plane, Phys. Fluids, 19 058101-058105, doi:10.1063/1.2722242.
Savage, S. B., and K. Hutter (1991), The dynamics of avalanches of granular materials from initiation to runout. part 1: Analysis, Acta Mech., 86(1-4), 201-223, doi:10.1007/BF01175958

Sibson, R. H. (1973), Interactions between temperature and pore fluid pressure during earthquake faulting: A mechanism for partial or total stress relief, Nat. Phys. Sci., 243, 66-68.

Sonder, I., B. Zimanowski, and R. Buttner (2006), Non-Newtonian viscosity of basaltic magma, Geophys. Res. Lett., 33, L02303, doi:10.1029/ 2005 GL024240.

Staron, L., and E. J. Hinch (2007), The spreading of a granular mass: Role of grain properties and initial conditions, Granular Matter, 9, 205-217, doi:10.1007/s10035-006-0033-z.

Strom, A. L. (2006), Morphology and internal structure of rockslides and rock avalanches: Grounds and constraints for their modelling, in Landslides From Massive Rock Slope Failure, Earth Environ. Sci. Ser. vol. 302, edited by S. G. Evans et al., pp. 243-266, Springer, Dordrecht, Netherlands.

Taberlet, N., P. Richard, J. T. Jenkins, and R. Delannay (2007), Density inversion in rapid granular flows: The supported regime, Eur. Phys. J. E, 22, 17-24, doi:10.1140/epje/e2007-00010-5

Taboada, A., K.-J. Chang, and J. Malavieille (2005a), Observations and simulation of force transmission in deformed conglomerates (Jiu-Jiu Fon, Taiwan), in Powders and Grains: 5th International Conference on Micromechanics of Granular Media, Stuttgart, vol. 1, edited by R. GarcíaRojo, H. J. Herrmann, and S. McNamara, pp. 73-75, A. A. Balkema, Brookfield, Vermont.

Taboada, A., K.-J. Chang, F. Radjaï, and F. Bouchette (2005b), Rheology, force transmission, and shear instabilities in frictional granular media from biaxial numerical tests using the contact dynamics method, J. Geophys. Res., 110, B09202, doi:10.1029/2003JB002955.

Taboada, A., N. Estrada, and F. Radjaï (2006), Additive decomposition of shear strength in cohesive granular media from grain-scale interactions, Phys. Rev. Lett., 97(9), 098302-098306, doi:10.1103/PhysRevLett. 97.098302 .

Vardoulakis, I. (2002a), Steady shear and thermal run-away in clayey gouges, Int. J. Solids Struct., 39(13), 3831-3844, doi:10.1016/S00207683(02)00179-8

Vardoulakis, I. (2002b), Dynamic thermo-pore mechanical analysis of catastrophic landslides, Géotechnique, 52(3), 157-171.

Veveakis, E., I. Vardoulakis, and G. Di Toro (2007), Thermoporomechanics of creeping landslides: the 1963 Vaiont slide, northern Italy, J. Geophys. Res., 112, F03026, doi:10.1029/2006JF000702.

Whittington, A., P. Richet, Y. Linard, and F. Holtz (2001), The viscosity of hydrous phonolites and trachytes, Chem. Geol., 174, 209-223.

Wibberley, C. A. J., and T. Shimamoto (2005), Earthquake slip weakening and asperities explained by thermal pressurization, Nature, 436, 689692, doi:10.1038/nature03901.

N. Estrada and A. Taboada, UMR 5243, Géosciences Montpellier, Université Montpellier II, INSU, CNRS, CC 60, Place East Bataillon, F-34095 Montpellier CEDEX 5, France. (nicolas.estrada@gm.univ-montp2.fr; alfredo.taboada@gm.univ-montp2.fr) 